\title{
Personalized Voice Activated Grasping System for a Robotic Exoskeleton Glove
}

\author{
Yunfei Guo \\ Thesis submitted to the Faculty of the \\ Virginia Polytechnic Institute and State University \\ in partial fulfillment of the requirements for the degree of \\ Master of Science \\ in \\ Computer Engineering
}

Pinhas Ben-Tzvi, Chair

Yunhui Zhu

Ryan M Gerdes

Dec 10, 2020

Blacksburg, Virginia

Keywords: Exoskeleton glove, Human Machine Interfacing, Embedded System, Voice activation, Speaker Verification.

Copyright 2021, Yunfei Guo 


\section{Personalized Voice Activated Grasping System for a Robotic Ex- oskeleton Glove}

Yunfei Guo

\section{(ABSTRACT)}

Controlling an exoskeleton glove with a highly efficient human-machine interface (HMI), while accurately applying force to each joint remains a hot topic. This paper proposes a fast, secure, accurate, and portable solution to control an exoskeleton glove. This state of the art solution includes both hardware and software components. The exoskeleton glove uses a modified serial elastic actuator (SEA) to achieve accurate force sensing. A portable electronic system is designed based on the SEA to allow force measurement, force application, slip detection, cloud computing, and a power supply to provide over 2 hours of continuous usage. A voice-control-based HMI referred to as the integrated trigger-word configurable voice activation and speaker verification system (CVASV), is integrated into a robotic exoskeleton glove to perform high-level control. The CVASV HMI is designed for embedded systems with limited computing power to perform voice-activation and voice-verification simultaneously. The system uses MobileNet as the feature extractor to reduce computational cost. The HMI is tuned to allow better performance in grasping daily objects. This study focuses on applying the CVASV HMI to the exoskeleton glove to perform a stable grasp with force-control and slip-detection using SEA based exoskeleton glove. This research found that using MobileNet as the speaker verification neural network can increase the speed of processing while maintaining similar verification accuracy. 


\title{
Personalized Voice Activated Grasping System for a Robotic Ex- oskeleton Glove
}

\author{
Yunfei Guo
}

(GENERAL AUDIENCE ABSTRACT)

The robotic exoskeleton glove used in this research is designed to help patients with hand disabilities. This thesis proposes a voice-activated grasping system to control the exoskeleton glove. Here, the user can use a self-defined keyword to activate the exoskeleton and use voice to control the exoskeleton. The voice command system can distinguish between different users' voices, thereby improving the safety of the glove control. A smartphone is used to process the voice commands and send them to an onboard computer on the exoskeleton glove. The exoskeleton glove then accurately applies force to each fingertip using a force feedback actuator.This study focused on designing a state of the art human machine interface to control an exoskeleton glove and perform an accurate and stable grasp. 


\section{Acknowledgments}

I would like to thank the ECE department, Professor Mark Jones, and Professor Paul Plassman for funding me as TA through my Master's degree.

I would also like to thank Professor Pinhas Ben-Tzvi, Wenda Xu, Sarthark Pradhan, Hailin

Ren, Yujiong Liu, and Taylor Njaka who provided valuable advice and greatly assisted me in this research.

I would also like to gratefully acknowledge the support of NVIDIA Corporation with the donation of the Titan Xp GPU.

Research reported in this publication was supported by the Eunice Kennedy Shriver National Institute of Child Health \& Human Development of the National Institutes of Health under Award Number R21HD095027. The content is solely the responsibility of the authors and does not necessarily represent the official views of the National Institutes of Health. 


\section{Contents}

List of Figures $\quad$ viii

List of Tables $\quad$ x

1 Introduction $\quad 1$

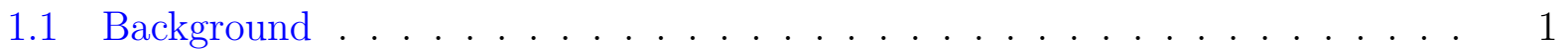

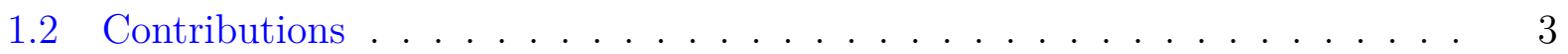

1.3 Thesis Structure . . . . . . . . . . . . . . . . . . 3

1.4 Selected Publications . . . . . . . . . . . . . . . . . 4

1.4.1 Peer-Reviewed Journal Paper . . . . . . . . . . . . . . . 4

1.4.2 Peer-Reviewed Conference Paper . . . . . . . . . . . . . 5

2 Literature Review $\quad 6$

2.1 Detecting intention to grasp during reaching movements from EEG . . . . . 6

2.2 Eyes are faster than hands: A soft wearable robot learns user intention from the egocentric view .......................... 7

2.3 Towards the development of a voice-controlled exoskeleton system for restoring hand function . . . . . . . . . . . . . . . 7

2.4 VGG-M Speaker Verification . . . . . . . . . . . . . . 8 
2.5 MobileNet . . . . . . . . . . . . . . . . . . . . . . 9

2.6 Intelligent Object Grasping and Learning System . . . . . . . . . . . . 10

3 Problem Statement and Proposed Solution 11

3.1 HMI Selection . . . . . . . . . . . . . . . . . . . . . . . . . . . 11

3.2 Hardware and Low-Level Control . . . . . . . . . . . . . . . . . . . . . 12

4 Hardware Design and Low-Level Control 14

4.1 Mechanical Design: SEA and RML Glove . . . . . . . . . . . . . . . 14

4.2 Electronics Design and Low Level Control . . . . . . . . . . . . . . . . 16

5 Personalized Voice Activated Grasping System 19

$5.1 \quad$ CVASV HMI . . . . . . . . . . . . . . . . . . . . . . . . . . 19

5.1 .1 Configurable Voice Activation . . . . . . . . . . . . . . . . . . . 21

5.1 .2 Voice Verification . . . . . . . . . . . . . . . . . . . . . 24

6 Experimental Setup and Results $\quad 27$

6.1 Electronics . . . . . . . . . . . . . . . . . . . . . . . . . . 27

6.2 Slip Detection and Low-Level Control . . . . . . . . . . . . . . . . . . 27

6.3 Neutral Network Feature Extractor in CVASV HMI . . . . . . . . . . . . . . 29

6.4 CVASV HMI . . . . . . . . . . . . . . . . . . . . . . . . 33

6.4.1 RML Exoskeleton Voice Control Dataset . . . . . . . . . . . . . . . . 34 
6.4.2 CVASV HMI Performance . . . . . . . . . . . . . . 35

6.5 Personalized Voice Activated Grasping System . . . . . . . . . . . . . . 38

7 Conclusion and Future Work 41

7.1 Conclusion . . . . . . . . . . . . . . . . . . 41

7.2 Future Work .......................... 42

7.2.1 Electronics and Hardware . . . . . . . . . . . . . . . 42

7.2.2 CVASV Recognition Subsystem . . . . . . . . . . . . . . 42

7.2.3 CVASV Verification Subsystem . . . . . . . . . . . . . 44

$\begin{array}{ll}\text { Bibliography } & 45\end{array}$ 


\section{List of Figures}

1.1 Novel Design of the latest RML glove with SEA and Electronics . . . . . . 2

4.1 Sample of Linear SEA on RML Glove . . . . . . . . . . . . . . . . 15

4.2 Design of the Rotatory SEA on the RML Glove . . . . . . . . . . . . 16

4.3 Structure of the Low-Level Control . . . . . . . . . . . . . . . . . 17

4.4 Electronics Overview . . . . . . . . . . . . . . . . . . . . . . . . . 18

5.1 Personalized Voice Activated Grasping System Overview . . . . . . . . . . . 20

5.2 Structure of CVASV HMI . . . . . . . . . . . . . . . . 21

5.3 Noise Filter . . . . . . . . . . . . . . . . . . . . . . . . . . 22

5.4 Flowchart of Loudness Detection . . . . . . . . . . . . . 23

5.5 Training and Testing with Modified MobileNetV1. (A) Training Add-on. (B) Testing Add-on . . . . . . . . . . . . . . . . . 26

6.1 Manufactured Electronics: (Green) Side mount computational unit and motor control unit. (Red) Power conversion unit with battery. . . . . . . . . . . . 28

6.2 Slip Detection Using Linear SEA . . . . . . . . . . . . . . . . . 30

6.3 Slip Detection Using Rotatory SEA . . . . . . . . . . . . . . . . . 31

6.4 (A) Modified MobileNetV1 Training and Validation Accuracy; (B) Modified MobileNetV1 Training and Validation Loss . . . . . . . . . . . . . . . 
6.5 (A) VGG-M Training and Validation Accuracy; (B) VGG-M Training and Validation Loss . . . . . . . . . . . . . . . . . . . . . 32

6.6 Structure of the Speaker Model of the RML Exoskeleton Voice Control Dataset 34

6.7 Grasping with Personalized Voice Activated Grasping System using Linear SEA 39

6.8 Grasping with Personalized Voice Activated Grasping System using Rotatory $\mathrm{SEA} \ldots \ldots \ldots \ldots \ldots \ldots \ldots \ldots$ 


\section{List of Tables}

6.1 Latency in Low Level Control of the Personalized Voice Activated Grasping System . . . . . . . . . . . . . . . . . . . . . . . 28

6.2 Comparison between Modified MobileNetV1 and VGG-M . . . . . . . . . . 31

6.3 CVASV HMI Accuracy Test . . . . . . . . . . . . . . . . . . . . 36

6.4 Time Cost to process a one audio command . . . . . . . . . . . . 36

6.5 Influence of Background Noise on CVASV HMI . . . . . . . . . . . . . . 36

6.6 Influence of Gender on CVASV HMI . . . . . . . . . . . . . . . . . 37

6.7 Influence of Accent on CVASV HMI . . . . . . . . . . . . . . . . . 38

6.8 Cosine distance between different commands of same speaker . . . . . . . . 38 


\section{List of Abbreviations}

ADL: Activities of Daily Living

API: Application Programming Interface

CNN: Convolution Neural Network

CVASV: Configurable Voice Activation and Speaker Verification

DOF: Degree of Freedom

FSR: Force Sensing Resistor

GMM: Gaussian Mixture Model

HMI: Human Machine Interface

KNN: K Nearest Neighbour Search

PCB: Printed Circuit Board

RML glove: Robotic Exoskeleton designed in Robotics and Mechatronics Lab at Virginia Tech

SEA: Serial Elastic Actuator 


\section{Chapter 1}

\section{Introduction}

\section{$1.1 \quad$ Background}

According to statistical data published in 2010, over 6.7 million of U.S. adults have difficulty grasping or handling small objects [1]. To potentially better the lives of such a large group of people, a robotic exoskeleton glove is designed to be used as a rehabilitation device for Activities of Daily Living (ADL) [2]. This medical robotic exoskeleton glove is designed in the Robotics and Mechatronics Lab (RML) at Virginia Tech. Therefore, the exoskeleton glove is named RML glove. The design of RML glove is inspired by motion sensing and hand modeling haptic exoskeleton glove $[3,4,5]$.

There are three generations of RML glove. The first generation is design by Ma, et al. It used cable transmission and force sensing resistor (FSR) to perform force feedback [6]. The first generation RML glove can be used for re-habitation therapy $[7,8]$. Lee, et al, added a slip detection, motion amplification system to improve the stability of the grasp [9]. Lee, et al, also designed an alternative version of RML glove to used as rehabilitation device [10].

Refour, et al, designed the second generation of the RML glove [11], which examined the feasibility of using articulated and coupled rigid linkages to transmit forces to the finger tips. A different version is design and implemented for infant medical rehabilitation [12].

Refour, et al, replaced pneumatic actuators used in the previous generation RML glove 


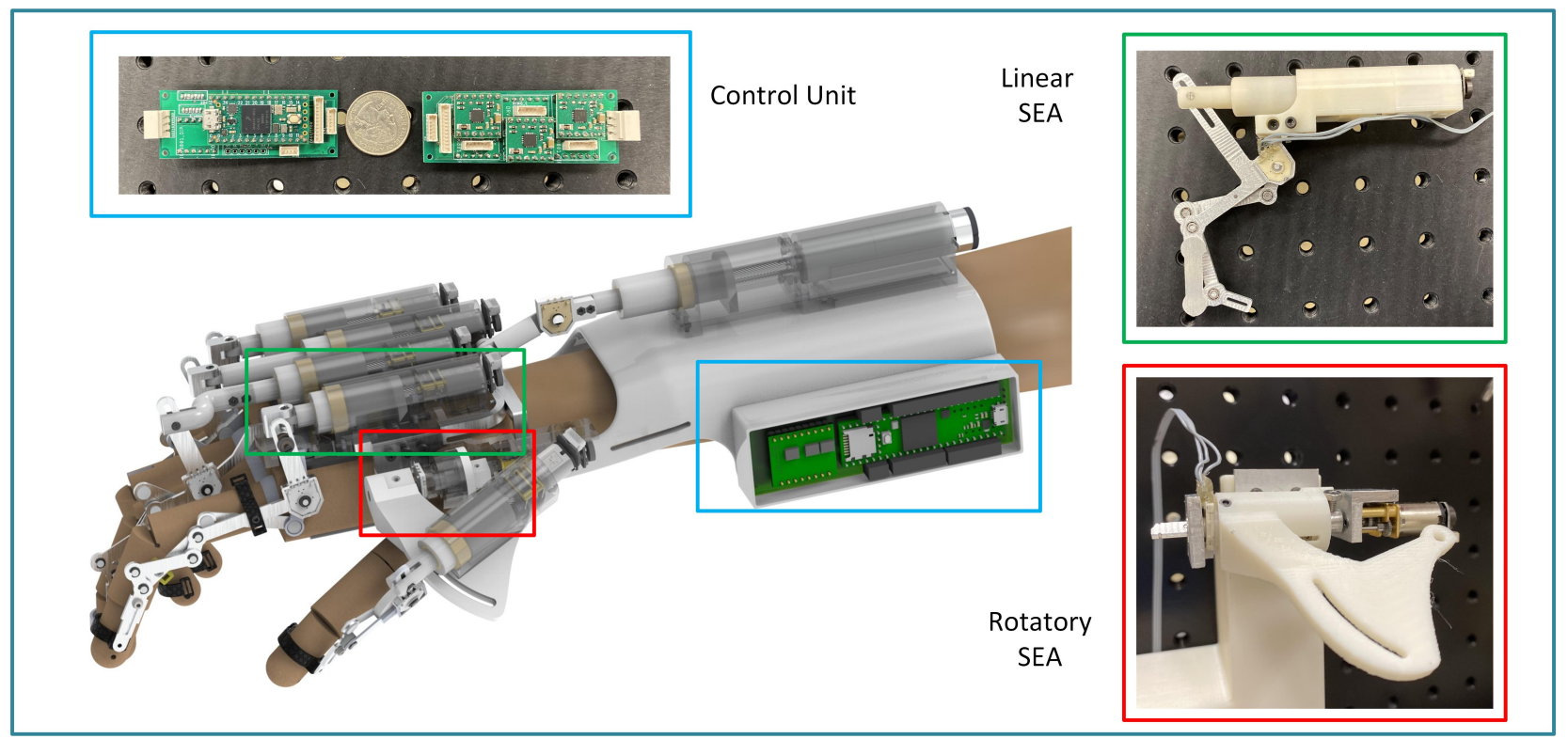

Figure 1.1: Novel Design of the latest RML glove with SEA and Electronics

with serials elastic actuators (SEA) and built the third generation of the RML glove [1] [13]. Chauhan, et al, proposed a grasp prediction algorithm to enhance the previous motion amplification system [14, 15]. Vanteddu, el al, improved the structure of the glove [2] and added deformation control for more stable grasping [16].

The latest generation RML exoskeleton glove is based on a rigid linkage mechanism connected with a linear SEA on each finger, to control each of the linkage mechanisms [17]. A rotatory SEA is used to control the MCP joint of the thumb [18], and a linear SEA to control the wrist joint. Each finger can perform 3 degrees of freedom (DOF) motion and exert 10N force through the linkage mechanism. The RML glove can perform the following eight basic grasps: Cylindrical Wrap, Sphere, Two Finger Pinch, Prismatic Small Stick, Index Extension Wrap, Disk, and Flat Parallel [14].

The design principle of the RML glove makes it easily wearable and portable. Compared to the previous electronics design of the RML glove [2], all electronics are integrated on a Print Circuit Board (PCB) with a separate power supply. The new electronics design is 
completely wearable when incorporated into the RML glove. The new design contains a faster micro-controller with a Bluetooth model, allowing for cloud computing. The PCB is designed to be have three separate smaller PCBs for an integrated battery, a high precision angular sensor, a power converter, and an over-volt protection circuit. This thesis details the designs and provides diagrams of the electronics.

\subsection{Contributions}

In this study, two algorithms were developed. First, the Configurable Voice Activation and Speaker Verification algorithm was developed to control the RML glove and was tested on the glove prototype. Second, slip-detection algorithms were implemented using SEAs instead of force sensing resistors (FSRs).

The major contributions are summarized as follows:

1: Test the configurable voice activation and speaker verification algorithm using a public data set in order to test the feasibility of the selected neural network.

2: Implement the low-level force feedback control system into the RML glove. Design and manufacture the electronics and SEA.

3: Implement force detection using SEA and test the slip-detection algorithm.

4: Combine the low-level control system with the high-level intelligence voice control system. Test the complete system with one finger on the SEA.

\subsection{Thesis Structure}

A brief overview of each chapter is listed as follows: 
Chapter 1: Introduces the RML exoskeleton glove and provides a discussion on the advantages and disadvantages of each generation of the RML exoskeleton glove, and outlines the contributions of this research.

Chapter 2: Provides a literature review regarding the exoskeleton control method and related methods. It also provides background information about voice verification.

Chapter 3: States the current problem of controlling the RML exoskeleton glove and proposes a voice-based, accurate, fast solution to solve the current problem.

Chapter 4: Describes the hardware design of the SEA and electronics, as well as the lowlevel force control system and slip-detection algorithm.

Chapter 5: Describes the high-level voice command system.

Chapter 6: Shows the results of all the experiments that were conducted. It also provides a discussion on the experimental results.

Chapter 7: Summarizes the work and proposes potential future improvements.

\subsection{Selected Publications}

Disclosure: Contents from these publications are used in this thesis.

\subsubsection{Peer-Reviewed Journal Paper}

1: Guo, Y., Xu, W., Pradhan, S., Bravo, C.J., Ben-Tzvi, P., "Personalized Voice Activated Grasping System for Exoskeleton Glove" IFCA Mechatronics, pending submission. 


\subsubsection{Peer-Reviewed Conference Paper}

1: Xu, W., Pradhan, S., Guo, Y., Bravo, C.J., Ben-Tzvi, P., "Integrated and Configurable Voice Activation and Speaker Verification System for a Robotic Exoskeleton Glove", Proceedings of the 2020 ASME IDETC/CIE, 44th Mechanisms \& Robotics Conference, St. Louis, MO, Aug. 16-19, 2020.

2: Xu, W., Pradhan, S., Guo, Y., Bravo, C.J., Ben-Tzvi, P., "A Novel Design of a Robotic Glove System for Patients with Brachial Plexus Injuries", Proceedings of the 2020 ASME IDETC/CIE, 44th Mechanisms \& Robotics Conference, St. Louis, MO, Aug. 16-19, 2020. 


\section{Chapter 2}

\section{Literature Review}

In order to control the RML glove, the command system needs to be convenient, accurate, secured, and computationally efficient. A highly-efficient user-detection interface must be designed to control the exoskeleton glove system. To fulfil the aforementioned requirements, researchers have previously designed different types of user-interfaces for the exoskeleton device. These different methods require minimal limber movements.

\subsection{Detecting intention to grasp during reaching move- ments from EEG}

L. Randazzo, et al. [19] proposed an electroencephalogram (EEG)-based intention detector. The change in slow cortical potentials (SCPs) can be detected using electroencephalographics (EEGs). An SPC signal peaks approximately one second before the initiation of movements [19]. By detecting the SPC signal, the intention to grasp can be detected within 400ms with an accuracy greater than $70 \%$. The advantage of using a brain-computer interface is that the user can naturally control the exoskeleton with low latency. However, using EEG requires the user to wear a large EGG device, and the accuracy (above 70\%) of EEG-based detection is still relatively low. It is not portable, and the accuracy does not fulfill our requirements. 


\subsection{Eyes are faster than hands: A soft wearable robot learns user intention from the egocentric view}

Daekyum Kim, et al. [20] proposed a vision-based intention detector. Here, the user needs to wear glasses with an inbuilt camera; the camera shares the same view of the user and uses it to detect their intention. This system also has a moderate response speed of 300$800 \mathrm{~ms}$. However, multiple objects in the camera or occlusions between objects will obstruct the system's overall performance.

\subsection{Towards the development of a voice-controlled ex- oskeleton system for restoring hand function}

Xuefeng Wang, et al. [21] proposed using Apple's Siri to control their exoskeleton glove, with the program running on an iPhone. According to Apple, Siri has a relatively low (5\%) word error rate and is able to run without noticeable latency on an iPhone. It is proven to be robust and stable by many Siri users. The lack of configurable activation keyword is inconvenient when using the glove in public. However, the lack of speaker verification can cause security related problems.

Compared with EEG and vision detection methods discussed above, voice control does not have major drawbacks except for user identification. The following research is concerned with speaker verification which can possibly solve the user identification issue. 


\subsection{VGG-M Speaker Verification}

VGG-M with Softmax loss function is a deep learning approach to the voice verification system proposed by Nargrani et al [22]. This deep-learning voice verification system achieves better accuracy $(10.2 \%)$ than the non-deep learning baseline (15.0\%).

The voice verification system consists of three sections. The first section is the prepossessing section, where each audio file is divided into several 3-second audio clips. Each audio clip is turned into a 512x300 spectrum using Fourier transform. The spectrum is treated as 2D images and fed into an utterance-level feature extractor.

The second section consists of utterance level feature extraction, where 1251 speakers are classified as 1251 classes by a deep neural network, and the last layer is removed. The remaining network is then used as a feature extractor. The VGG-M network is modified based on VGG16. Compared with VGG16, VGG-M can take various lengths of the 2D spectrum as an input. The output of the feature extractor is later used in the verification process.

The third section consists of the verification calculation. If the cosine of the distance between two different audio samples is within a threshold, the two samples are considered to be the same class. The baseline method changes the average pooling layer to match the test-time length so that the network can take inputs of various lengths. To make the verification more robust, the thesis also proposes a Test Time Augmentation 2 and Test Time Augmentation 3 method, which randomly select ten samples from the entire data and calculates the average distance between features. The VGG-M network with Softmax loss, Global Average Pooling, and Test Time Augmentation 2 were used as the deep learning baseline in this research. 


\subsection{MobileNet}

When using personalized voice activation and command system on exoskeletons, computation speed is crucial. The networks proposed by Nagran et al. [22] are not the fastest networks to run on a mobile device. As such, Andrew et al. [23] proposes an efficient convolution neural network used on image classification. MobileNet uses a deep-wise separable convolution to replace the traditional convolution. $C_{\text {traditional }}$ : Computation cost of traditional convolution. The cost of a traditional convolution is shown in Eq. 2.1. The deep-wise separable convolution is shown in Eq.2.2. Compared to traditional convolution, the MobileNet's deep-wise separable convolution is faster based on Eq.2.3.

$$
\begin{gathered}
C_{\text {traditional }}=H_{i} \times W_{i} \times C_{i} \times H_{k} \times W_{k} \times C_{o} \\
C_{\text {mobilenet }}=H_{i} \times W_{i} \times C_{i} \times H_{k} \times W_{k}+C_{i} \times C_{o} \times H_{i} \times W_{i} \times W_{k} \\
\frac{C_{\text {mobilenet }}}{C_{\text {traditional }}}=\frac{1}{C_{o}}+\frac{1}{H_{k} \times W_{k}}
\end{gathered}
$$

where $C_{\text {mobilenet }}$ is the computation cost of MobileNetV1, $H_{i}$ is the height of the input array, $W_{i}$ is the width of the input array, $C_{i}$ is the channel of the input array, $H_{k}$ is the height of the kernel, $W_{k}$ is the width of the kernel, and $C_{o}$ is the channel of the output array.

MobileNet-224 (MobileNetV1) achieves similar accuracy (70.6\%) compared to VGG16 (71.5\%) on ImageNet dataset. MobileNet-224 has far fewer parameters (4.2 million) than VGG16 (138 million); thus, it is faster than VGG16. The VGG-M network is modified based on VGG16, which has a similar accuracy in image classification. It is possible to use this 
method to accelerate the existing speaker verification process.

\subsection{Intelligent Object Grasping and Learning System}

Brielle [24] proposed an intelligent object grasping and learning system to control the previous version of the RML glove.

The electronics design contains a WiFi model and Teensy 3.2 micro-controller with no power supply. The size of the electronics is $5.3 \mathrm{~cm} \times 8 \mathrm{~cm} \times 4 \mathrm{~cm}$, which is not portable. The lowlevel control programs run in a state machine using a single thread which bottlenecks sensor readings. Therefore, the system experiences noticeable delays while controlling all fingers simultaneously.

Force sensors are placed on the fingertips to measure and regulate forces on the fingertips. The force sensors are also used to monitor any force changes on the fingertips. When a grasped object starts to slip, the force measured on the fingertips would inherently decrease dramatically. Therefore, if there is a dramatic decrease in the force-sensor reading, it indicates that the object, grasped by the user, is slipping. The HMI system uses the user's hand movement to initiate a grasp - the system is activated by sensing a minor twitch of the finger. Whenever the force sensing on the finger changes, grasp will be initiated. 


\section{Chapter 3}

\section{Problem Statement and Proposed}

\section{Solution}

In order to build an accurate, secured, portable RML glove grasping system, two major problems need to be solved. First, a good HMI needs to be designed such that it is more superior than other existing HMIs. Second, the electronics and hardware need to be updated to fit the HMI.

\subsection{HMI Selection}

The required design principles for the RML exoskeleton glove HMI include speed, accuracy, portability and security. Compared with EEG and vision detection methods discussed in the previous section, voice detection does not have major drawbacks. The accuracy is relatively high and the latency is moderate. Using voice control will be the most applicable and appropriate method to control the RML glove.

However, the problem with current voice control HMI is the lack of speaker verification

process. After the system is activated, there is no security check on the input command. If this system is used as the exoskeleton HMI, random people can control the exoskeleton glove while the user is wearing it. This will cause serious safety related problems to the wearer. 
Thus, this paper proposes a trigger-word configurable voice activation and speaker verification (CVASV) HMI, which enables fast and accurate command-input. With the CVASV HMI, the activation trigger-word can be configurable, and all commands can be verified on a platform with limited computational power. With the help of an application programming interface (API) from large companies such as Google and Apple, low computational cost configurable voice activation and recognition can be achieved. There is no available API for text-independent speaker verification. Nagran et al. [22] used a deep neural network to accomplish text-independent speaker verification and received a $2.87 \%$ error rate. Since VGG-M has fewer layers and parameters, it is faster than ThinResNet in terms of computational speed. However, VGG-M is not the fastest network that can be used on portable devices. In this thesis, MobileNetV1 [23] was used to build a text-independent speaker verification platform with limited computing power.

\subsection{Hardware and Low-Level Control}

In order to properly test the Personalized Voice Activated Grasping System, the hardware and low-level control had to be redesigned. The previous electronics were oversized, slow, and functionally incomplete. In contrast, the new electronics design focuses on making the electronics portable and faster by reducing the size and upgrading the micro-controller. A power supply section was added to make the electronics functionally complete.

The previous low-level control utilizes a single-threaded blocking control system. Simultaneously controlling the $7 \mathrm{SEAs}$ on this structure thereby induces considerable delays. The new control system introduces a FreeRTOS-based, multi-threading low-level force control system.

To implement the slip-detection system, previous research implemented slip detection by 
measuring the change of the normal force on each finger using force-sensing resistors (FSRs). Rather than using FSRs, SEAs can be used to measure the normal force when grasping an object indirectly without the need to place force sensors between the fingers and the object to be grasped. Thus, slip detection was re-implemented using SEAs.

The updated hardware and low-level control will ensure a stable and high efficient control of the RML exoskeleton glove. 


\section{Chapter 4}

\section{Hardware Design and Low-Level}

\section{Control}

This chapter introduces the design of the complete hardware system. The main two sections include mechanical design and electronics design. The mechanical design section introduces the SEA and the RML glove. The electronics design section introduces the electronic components and the low-level control architecture.

\subsection{Mechanical Design: SEA and RML Glove}

A Linear SEA and a rotatory SEA are shown in Fig. 1.1. Linear SEAs and articulated linkage mechanisms are used for all fingers on the RML glove. A linear SEA is used to perform indirect sensing of contact forces between the fingers and the grasped object. In Fig.4.1, the red enclosed box points to an angular potentiometer which can measure the angle of the linkage attached to the SEA. The distance denoted by AC can be calculated based on the angle measurement. The blue enclosed box points to a magnetic encoder which can measure the distance between points $\mathrm{C}$ and $\mathrm{E}$. There exists a spring between points $\mathrm{A}$ and $\mathrm{D}$, and $\mathrm{CD}$ and $\mathrm{CB}$ are of known value. The difference between $\mathrm{AE}$ and $\mathrm{DE}$ calculates the compressed spring length. The spring length calculation is shown in Eq. 4.1. Force can be calculated using Eq.4.2. 


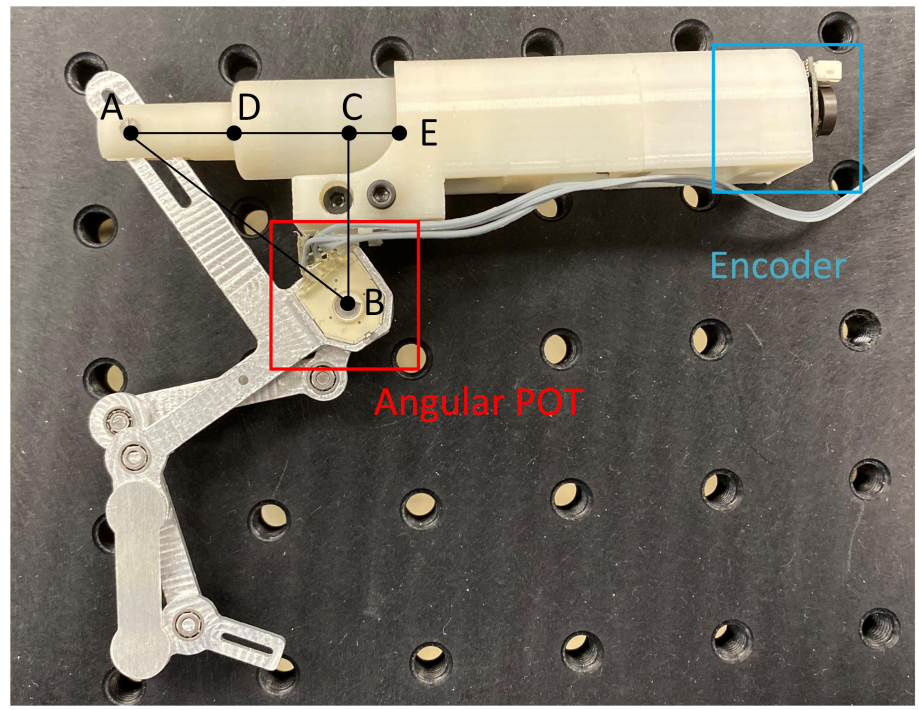

Figure 4.1: Sample of Linear SEA on RML Glove

$$
\begin{gathered}
L_{\text {spring }}=\cos (\angle A B C) \times C B+C E-D C-D E \\
\text { force }=k_{\text {spring }} \times \delta L_{\text {spring }}
\end{gathered}
$$

The rotatory SEA is designed to duplicate the motion of the metacarpophalangeal (MCP) joint. The Rotatory SEA uses a similar structure and uses a torsion spring instead of a coil spring for the linear SEA. Fig. 4.2 shows the design of a rotatory SEA. The red enclosed box highlights the output shaft, and the blue enclosed box highlights the input shaft. There exists a torsion spring between the output shaft and the input shaft. The force can be easily calculated by measuring the difference between the input shaft and output shaft angles. 


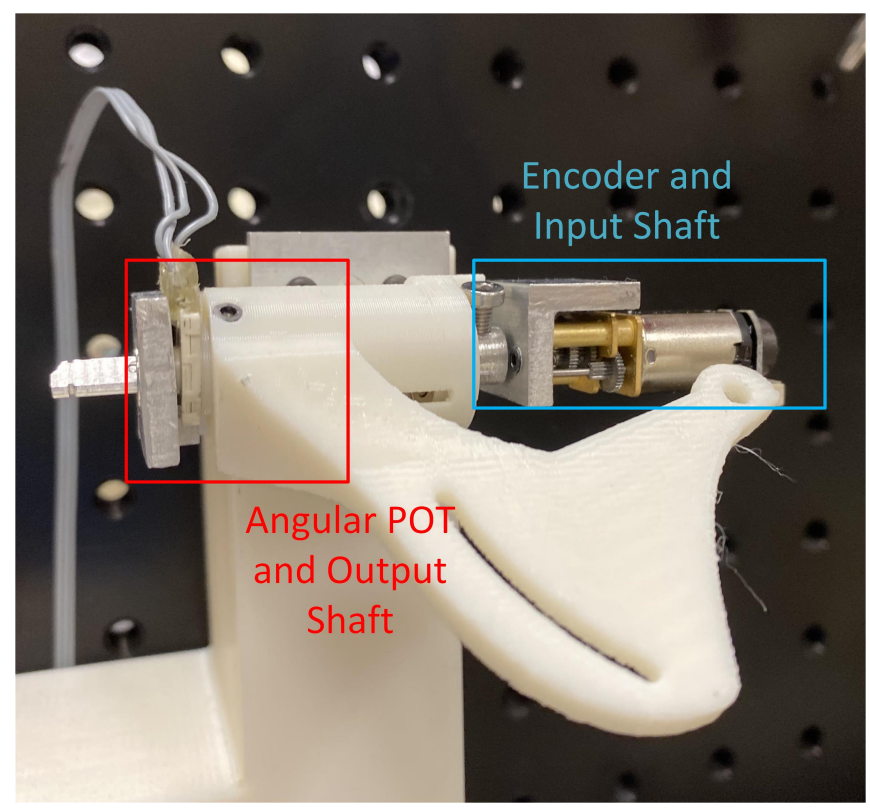

Figure 4.2: Design of the Rotatory SEA on the RML Glove

\subsection{Electronics Design and Low Level Control}

The electronics design of the RML glove is portable, has low latency, and modulated. All the electronics are embedded in the RML glove. The electronics design is modulated, and each part can be easily replaced. No cable connections are required to control the RML glove. The onboard micro-controller is responsible for sensor reading and performing low-level control, including force control and slip detection. The slip detection uses the same idea as Lee [24] proposed, in order to measure the force change on the fingertip to detect slip. Instead of using a force sensor, the SEA is used as the force sensing device. An integrated wearable battery box will supply power for two hours of continuous operation.

Pololu Gearmotor (380:1 gear ratio or 10000:1 gear ratio depending on the joint) is used for all SEAs to produce sufficient power and torque to actuate the RML glove. Encoders and an angular potentiometer are attached to measure each finger and wrist angle and calculate the force output of the SEAs. A micro-controller, called Teensy 4.0, was selected to read 


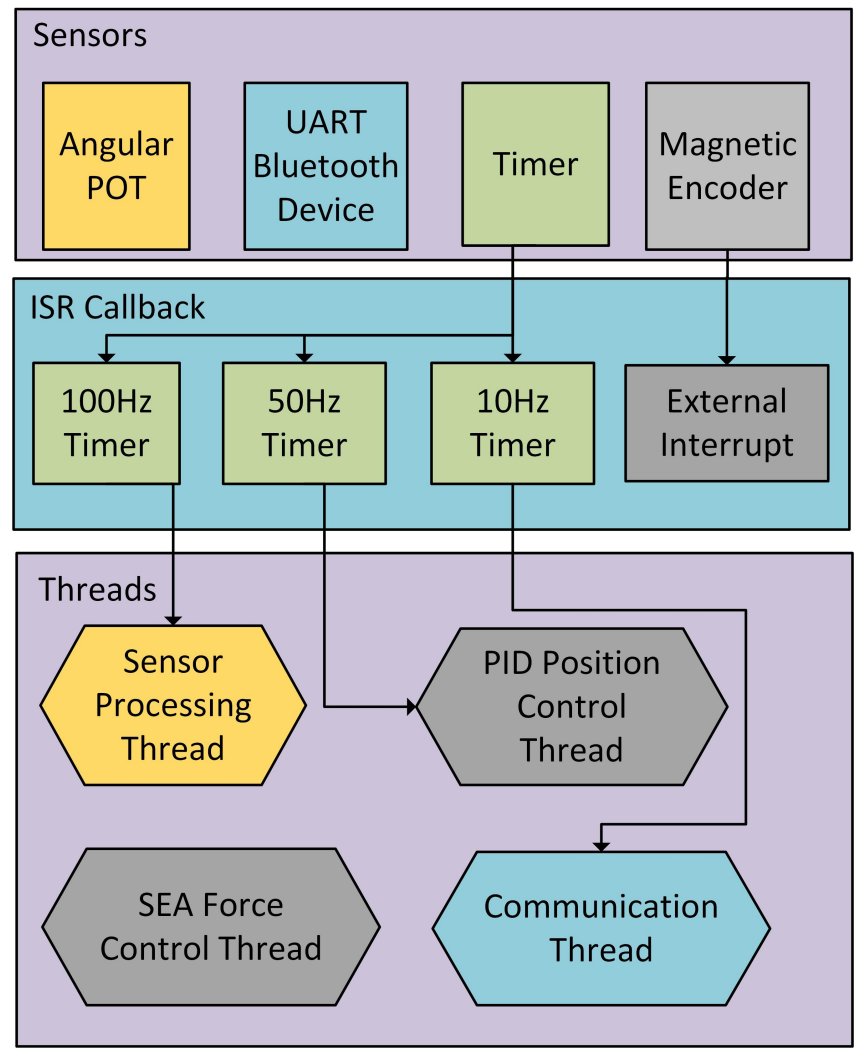

Figure 4.3: Structure of the Low-Level Control 


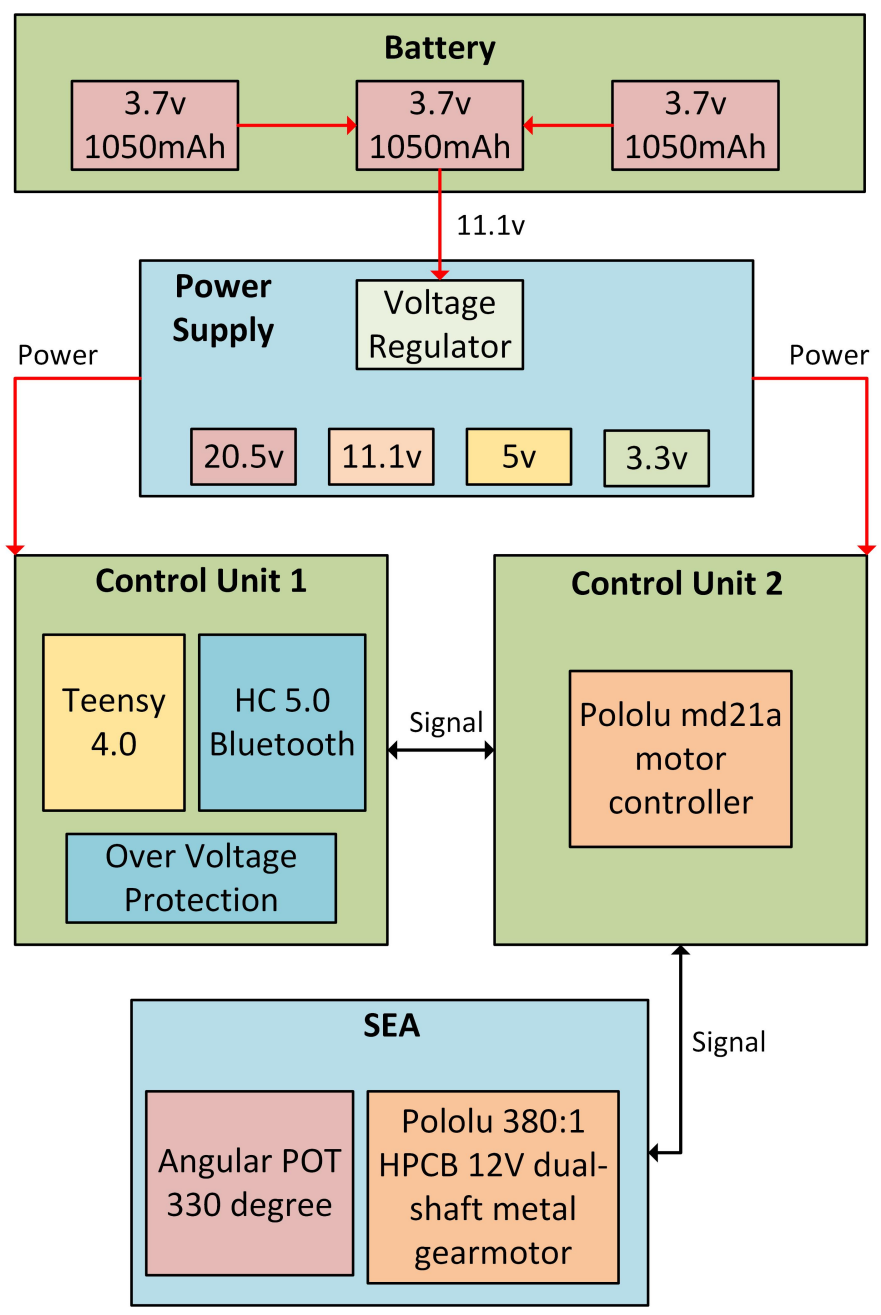

Figure 4.4: Electronics Overview

data from sensors and perform low-level control. A real-time system iwass used to minimize sensor reading latency and provide the ability to perform parallel computing. The structure of the low-level control system is shown in Fig 4.3.

Three separate PCBs were designed to place all the components on the computational box and power conversion. The components overview of each layer is shown in Fig. 4.4. 


\section{Chapter 5}

\section{Personalized Voice Activated}

\section{Grasping System}

The Personalized Voice Activated Grasping System shown in Fig. 5.1 contains a smartphone, a microphone, and a micro-controller. The microphone inputs raw data into the smartphone, and all the personalized voice activation and verification is calculated onboard. The microcontroller is placed on the exoskeleton to receive verified commands. The smartphone and micro-controller communicate through Bluetooth. The micro-controller is responsible for processing signals from sensors and perform force feedback control and slip detection algorithm. With minimal carry-on devices and cable connections, this system is designed to maximize portability and convenience of use.

\subsection{CVASV HMI}

The configurable personalized voice activation and command system can be divided into two sections. The first section controls the activation process, and the second section contains the verification process. Fig. 5.2 shows the thread assignments and the tasks that occur in each thread. The configurable voice activation section takes the raw audio data as input and outputs the accepted audio data. The microphone streaming callback continuously generates 0.5-second audio segments. The audio collection thread contains a noise reduction filter and 


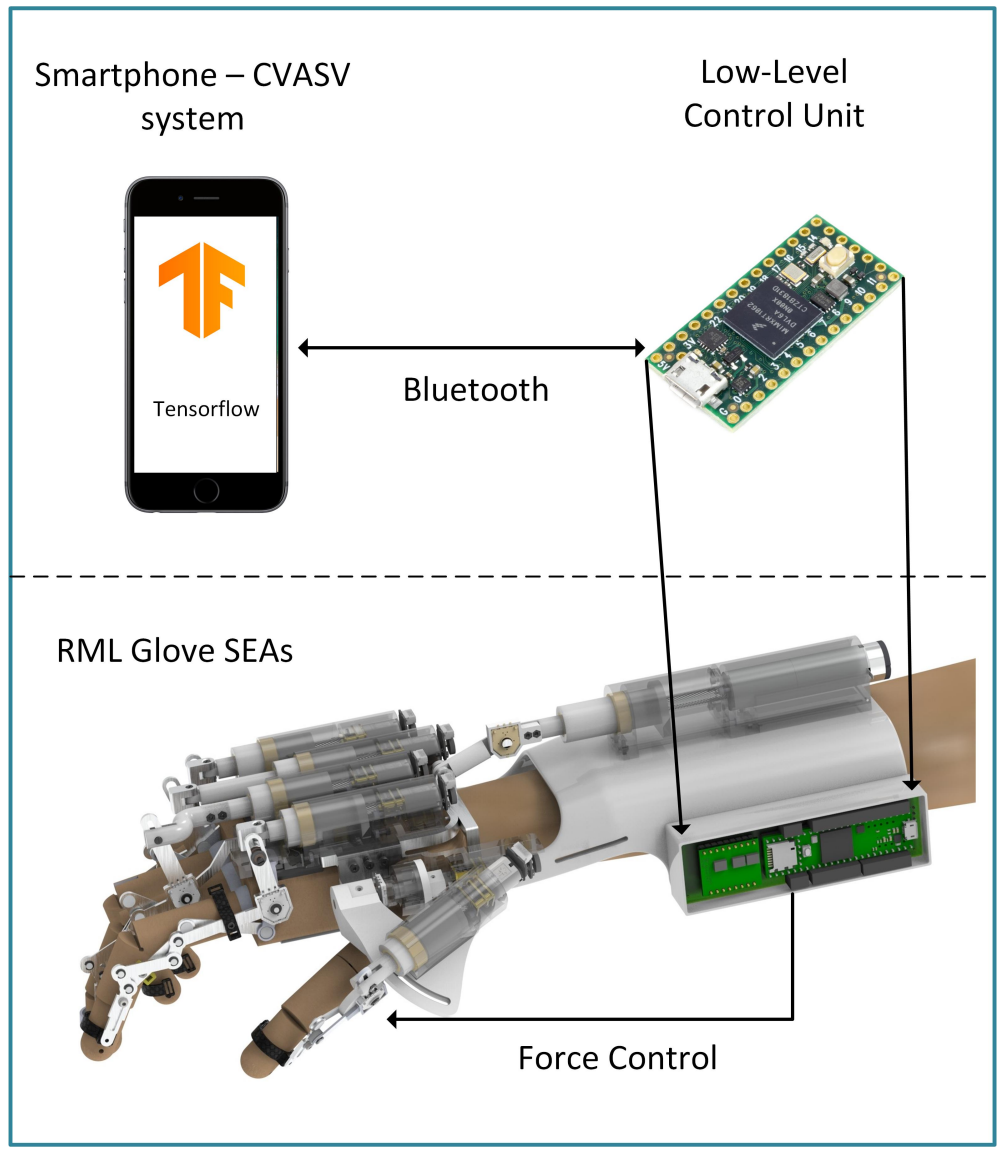

Figure 5.1: Personalized Voice Activated Grasping System Overview 


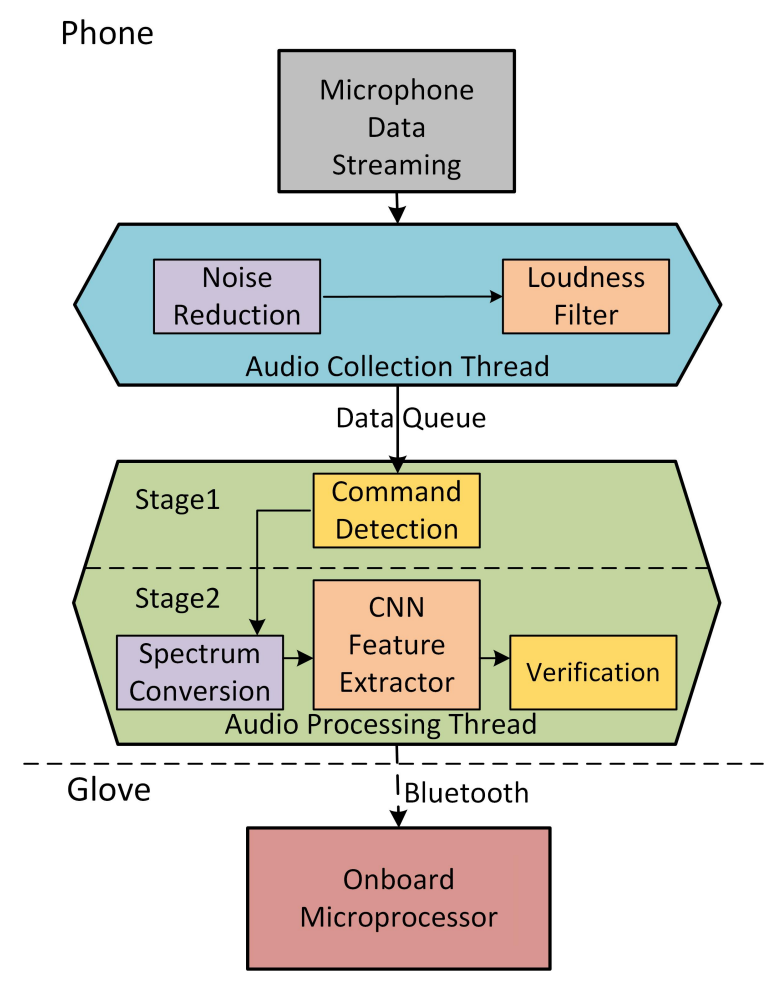

Figure 5.2: Structure of CVASV HMI

a loudness filter. If the loudness is greater than the threshold and a complete sentence has been detected, the audio collection thread then enters the pre-active mode. The audio data queue then sends data from the audio collection thread to the audio processing thread under pre-active mode. The voice processing thread consists of two stages. The command detection stage uses voice recognition API. After the command is accepted, it enters the speaker verification stage. The MobileNetV1 speaker verification system verifies if the audio belongs to one of the enrolled speakers.

\subsubsection{Configurable Voice Activation}

The voice activation system includes a noise reduction filter, a loudness filter, and a command detector. The system is designed to detect possible activation commands using minimal 


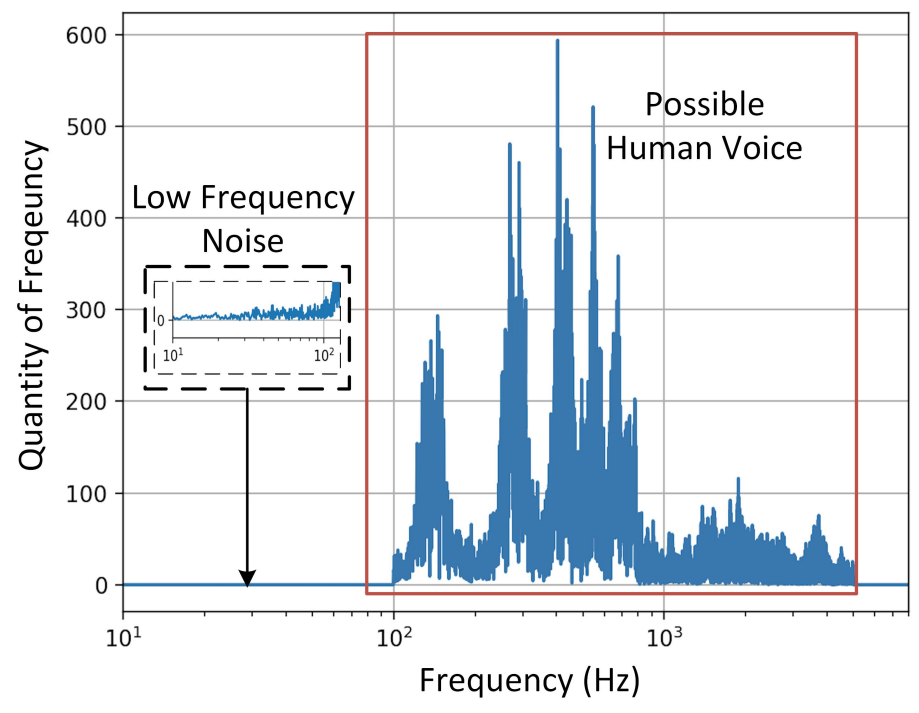

Figure 5.3: Noise Filter

computational cost. The system enters a pre-activated mode if the human voice's intensity is greater than a threshold. A human-like voice is checked using voice activation API to verify if an activation trigger-word is present. If the activation trigger-word is present, the system then enters into an activated mode, and the audio is passed to the voice verification system.

The raw input of the microphone voice contains high and low-frequency noise. With a noise reduction filter, the system will enter the pre-active mode less frequently from false-positive activations in a noisy environment.

A noise-reduction filter is used to minimize high and low-frequency noise. It converts raw input from the microphone to the time-independent frequency domain using a Fourier transform. The total relative power density within each frequency band is measured. Highfrequency and low-frequency bins are considered to be noise, and are subsequently trimmed from the spectrum. The time-independent Fourier transform [25] of the original audio data and the filtered audio data are shown in Fig. 5.3. The remaining frequencies contain potential human voices, which then enter the loudness detector. 


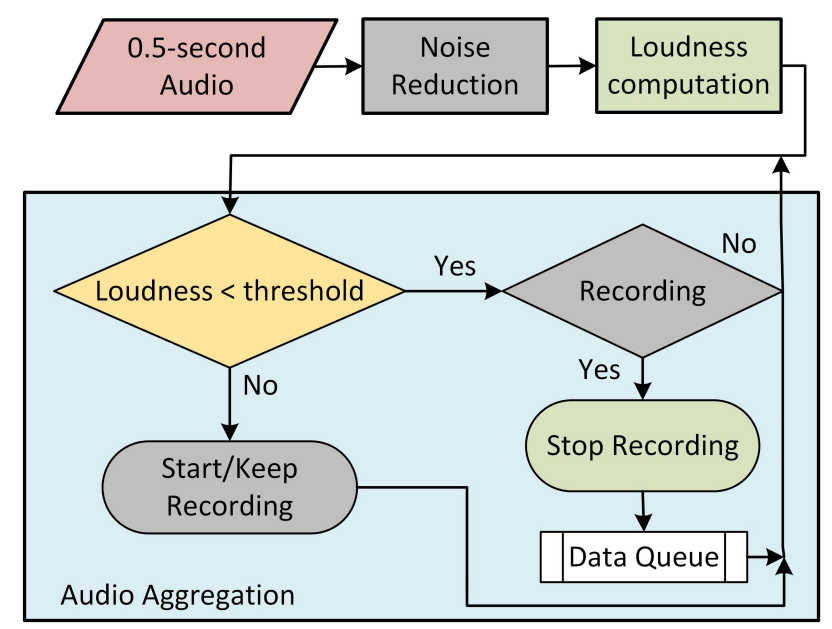

Figure 5.4: Flowchart of Loudness Detection

The loudness detector aggregates small audio segments into complete sentences. Audio data containing a loudness level above a certain threshold sets the system into pre-active mode. The input data is a 0.5 -second filtered audio segment. These audio segments are grouped into a larger audio segment based on the margin between each word. It was assumed that if the margin between words is longer than 0.5 -seconds, the sentence ends. Using this assumption, the loudness detector with audio aggregation is implemented according to the flowchart described in Fig. 5.4.

After the loudness detector detects a complete sentence, the audio data is sent to the audio processing thread for voice recognition. The loudness detector keeps monitoring the environment. In the voice processing thread, the complete sentence will be recognized using API. Voice recognition API from Amazon, Google, or Apple is proven to be accurate and requires low computational cost.

If the system is not activated, the API result is used for activating the trigger-word detection. But, if the system is already activated, the API result is instead forwarded directly as an unverified command. Words with similar pronunciation to the activation trigger-word or the command itself are accepted. After checking that the trigger-word or command is valid, 
speaker verification is ultimately performed using the filtered audio data.

\subsubsection{Voice Verification}

The voice verification method was improved based upon Nagrani et al. [22] VGG-M with the Softmax loss function speaker verification method. Instead of using VGG-M, the low computational cost network MobileNetV1 was used. The size of the MobileNetV1 was mod-

ified. The size of the network was reduced to $75 \%$ of the original network such that the alpha value equals 0.75 . The network was modified to adapt $2 \mathrm{D}$ spectrum input.

The training procedure for MobileNetV1 is similar to the baseline VGG-M method. Filtered audio data from the previous speaker activation section is converted into a 2D spectrum [25] with both frequency and time information. The 2D spectrum is fed into MobileNetV1. The speaker utterance is grouped using the Global Average Pooling (GAP) into 768 features. The 768 features are classified using a dense layer. The loss function used for classification is the standard Softmax loss function:

$$
P(y=j \mid x)=\frac{e^{X^{T} W_{j}}}{\sum_{k=1}^{K} e^{X^{T} W_{k}}}
$$

where $x$ is the spectrum input, $j$ is the label of a certain speaker, $X$ is the output of the feature from the neural network, $k$ is the number of classes, and $w$ is the weighting vector. The training process is shown in Fig. 5.5.

During the speaker verification procedure, the last dense layer of the MobileNetV1 is detached. The output of the network has 768 features. The distance between two audio features vector $A$ and $B$ is calculated. If the distance is less than an enrollment threshold, these two audios are from the same speaker. The verification procedure is shown in Fig. 
5.5. The distance between features is calculated by cosine of the distance [26]. The cosine distance $\left(D_{c}\right)$ is defined as:

$$
D_{c}=\frac{\sum_{i=1}^{n} A_{i} B_{i}}{\sqrt{\sum_{i=1}^{n} A_{i}^{2}} \sqrt{\sum_{i=1}^{n} B_{i}^{2}}}
$$

During the enrollment process, the user needs to record a $x$-second long enrollment audio. The audio is then randomly cut into $n$ audio segments each having a 3-second length. These $n$ audio clips are processed by the neural network and each audio clip is turned into a feature vector $(F)$. The average enroll-feature vector $\left(F_{\text {avg_enroll }}\right)$ can be calculated through Eq. 5.3.

$$
F_{a v g}=\frac{\sum_{k=1}^{n} F_{n}}{n}
$$

During the verification process, the input test audio is cut into $n$ audio segments, each having a 3-second length. The average test-feature vector $\left(F_{\text {avg_test }}\right)$ can also be calculated through Eq. 5.3.

The average distance between the same speaker and different speakers over a verification training data set can be calculated. The average distance between different speakers is used as the verification threshold. The verification training data set contains $K$ speakers. The threshold $(T)$ is chosen by calculating the mean distance between the average enroll-feature $\left(F_{\text {avg_enroll }}\right)$ and the average test-feature $\left(F_{\text {avg_test }}\right)$ over $K$ speakers, as shown in Eq. 5.4.

$$
T=\frac{\sum_{k=1}^{K} \operatorname{dist}\left(F_{\text {avg_enroll }}, F_{\text {avg_test }}\right)}{K}
$$




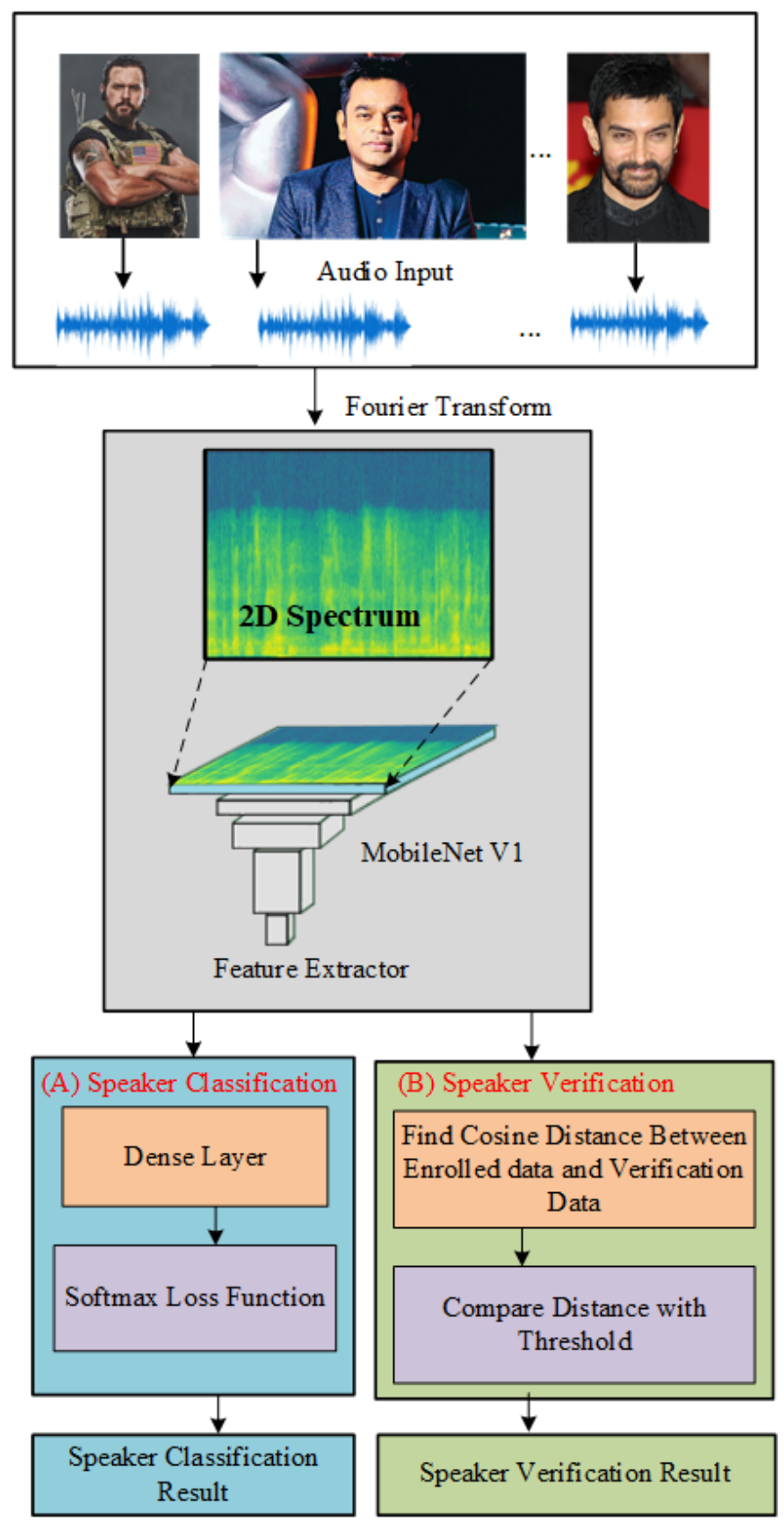

Figure 5.5: Training and Testing with Modified MobileNetV1. (A) Training Add-on. (B) Testing Add-on 


\section{Chapter 6}

\section{Experimental Setup and Results}

This chapter introduces the experimental setup and the results. The following components were tested: Electronics, low-level control, slip detection algorithm, CVASV HMI, and the complete RML glove grasping system. Each of these components is discussed in detail below.

\subsection{Electronics}

The electronics were built and tested. There are three separate electronics subsystems responsible for the onboard computation, motor control, and power conversion. The manufactured electronics are shown in Fig. 6.1. These electronics have been tested for functionality and have been running for over 100 hours without any issues. A 12V, 1.05 Amp-hour battery can continuously power both the electronics and one finger SEA for around 7 hours.

\subsection{Slip Detection and Low-Level Control}

The latency of the low-level control has been tested. Three major components can cause latency: sensor reading, SEA position control, and SEA force control. The micro-controller used is a Teensy $4.0 @ 800 \mathrm{MHz}$, and latency is measured for all three components. The results are provided in Table 6.1. The experiment demonstrated that the multi-threading system is well designed, where the low-level control only has a total of $2 \mathrm{~ms}$ latency caused 


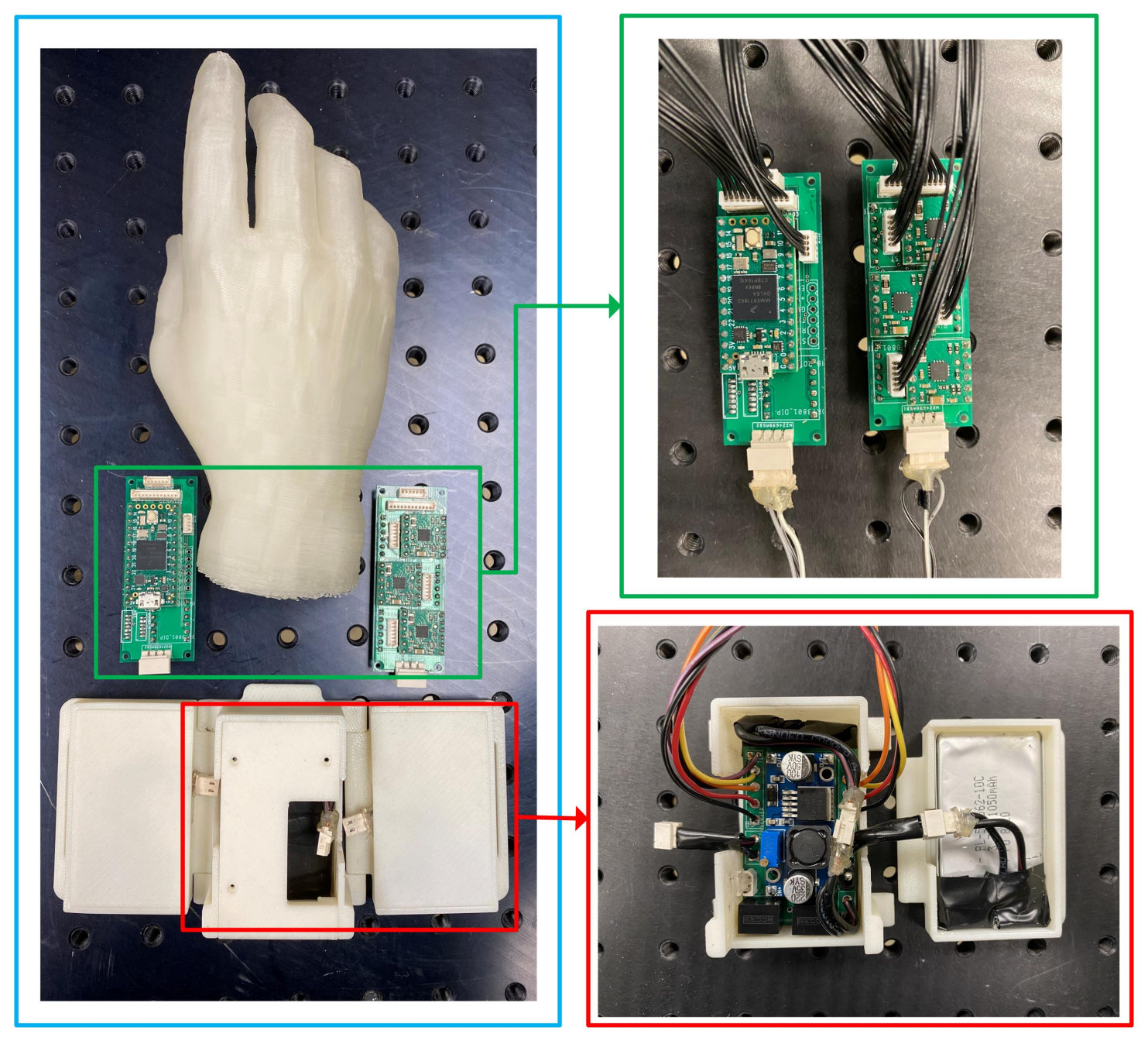

Figure 6.1: Manufactured Electronics: (Green) Side mount computational unit and motor control unit. (Red) Power conversion unit with battery.

Table 6.1: Latency in Low Level Control of the Personalized Voice Activated Grasping System

\begin{tabular}{c|c|c}
\hline \hline & Designed Time Cost & Latency \\
\hline Sensor Reading & $10 \mathrm{~ms}$ & $0 \mathrm{~ms}$ \\
SEA Position Control & $10 \mathrm{~ms}$ & $0 \mathrm{~ms}$ \\
SEA Force Control & $110 \mathrm{~ms}$ & $0 \mathrm{~ms}$ \\
Bluetooth Communication & $100 \mathrm{~ms}$ & $2 \mathrm{~ms}$ \\
\hline \hline
\end{tabular}

by Bluetooth communication.

According to Lee et al. [24], detecting the force differences between different fingertips can 
indicate slippage of the grasped object. In this experiment, instead of using a resistorbased force-sensor, an SEA was used to measure contact forces. One of the experiments demonstrated that a plastic bottle can be stably grasped with minimal force using a single finger SEA. The single finger SEA is manually rotated by 90 degrees and shaken until slip can be visually observed. During this process, spring compression vs. time is recorded. The results of the linear SEA readings are shown in Fig. 6.2, and the results of rotatory SEA readings are shown in Fig. 6.3. Spring compression is proportional to force; thus, the change in spring compression is proportional to the change in force. The SEA detects the slip and the low-level force feedback control can cause the SEA to apply the appropriate level of force to eliminate the slip.

\subsection{Neutral Network Feature Extractor in CVASV HMI}

The VoxCeleb1 [27] dataset is used for training the voice verification system in this research. This dataset contains 1251 celebrities giving presentations under different environments. There are over 100,000 utterances and 40 speakers are chosen for the verification test. The dataset contains noisy data which is suitable for training a robust speaker verification model.

The feature extractor training is performed on VoxCeleb1 dataset using MobileNetV1 and VGG-M. VGG-M will be used as a performance baseline. A 3-second audio clip is randomly

extracted from each audio file and converted to a 512x300 spectrum using Fourier transform. The 512x300 spectrum is used to train both MobileNetV1 and VGG-M networks. All networks will classify input audio data into 1251 classes.

The MobileNetV1 feature extractor (classification) was trained on the VoxCeleb1 dataset with 12 epochs. The training logs are shown in Fig. 6.4. To compare the performance between MobileNetV1 and VGG-M, the same data is trained on VGG-M and the results are 


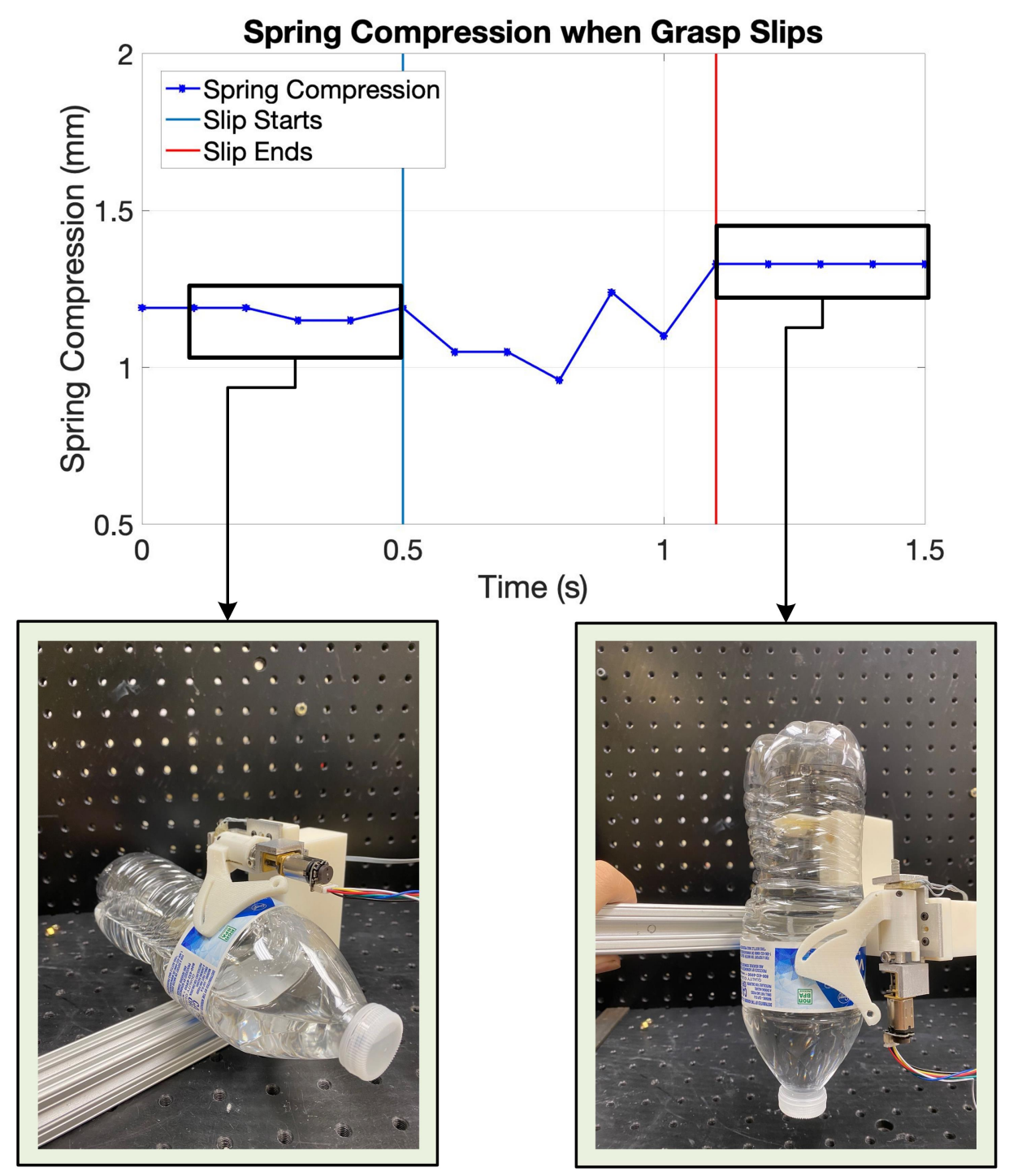

Figure 6.2: Slip Detection Using Linear SEA

shown in Fig. 6.5.

The performance comparison between the modified MobileNetV1 (MBN) and the VGG$\mathrm{M}$ is shown in Tab. 6.2. The modified MobileNetV1 achieves $18.6 \%$ higher accuracy in classification. The modified MobileNetV1 has $1.4 \%$ lower accuracy than VGG-M in the verification section. The use of MobileNetV1 as the feature extractor was $17.7 \%$ faster than using VGG-M. 


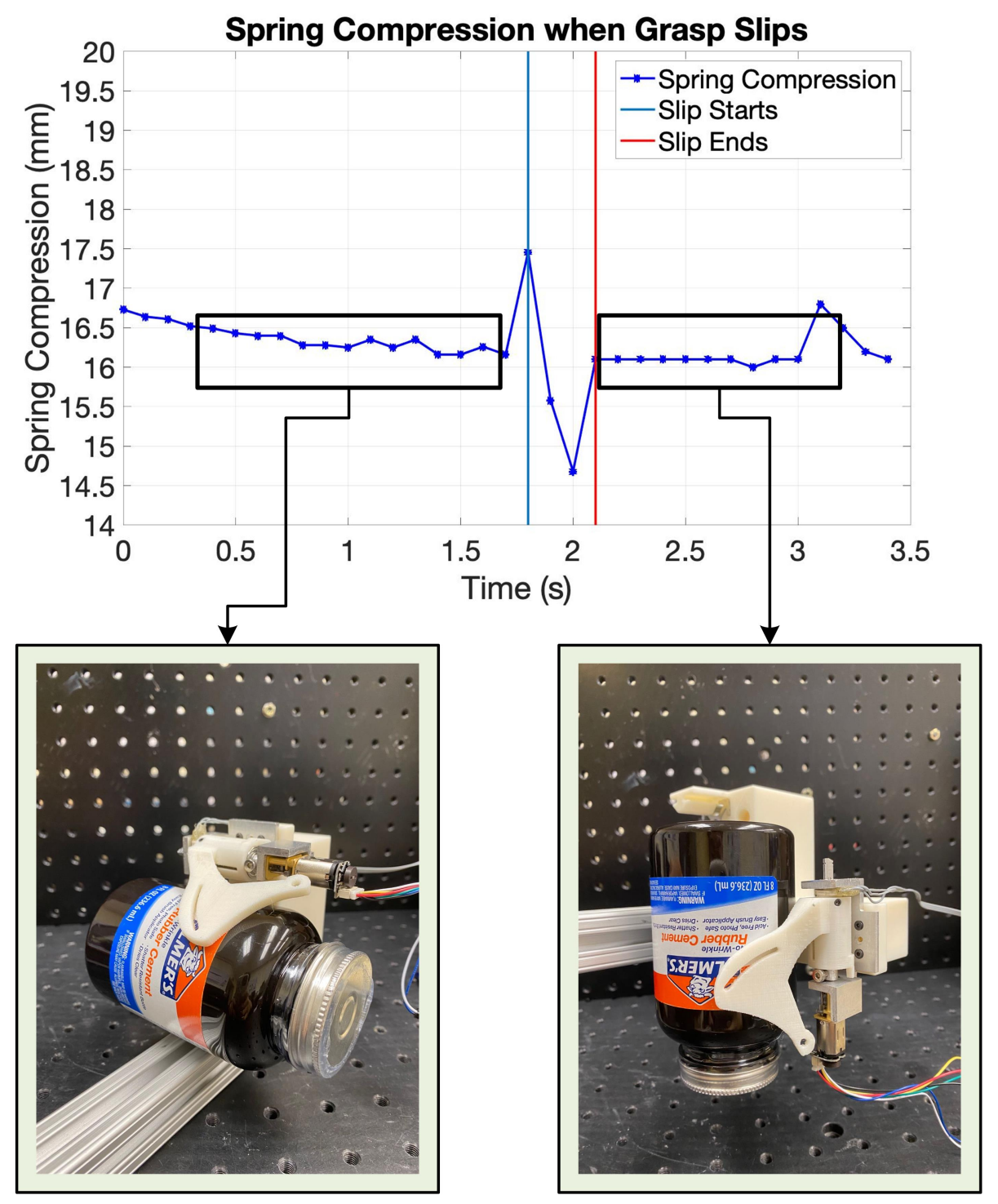

Figure 6.3: Slip Detection Using Rotatory SEA

Table 6.2: Comparison between Modified MobileNetV1 and VGG-M

\begin{tabular}{c|c|c}
\hline \hline & MBN & VGG-M \\
\hline Classification Accuracy & $\mathbf{7 9 . 8 \%}$ & $61.2 \%$ \\
Verification Accuracy $\left(D_{c}\right)$ & $88.9 \%$ & $\mathbf{9 0 . 3} \%$ \\
Time cost & $\mathbf{7 3 . 2 m s}$ & $88.9 \mathrm{~ms}$ \\
Total Parameters & $\mathbf{1 , 8 3 2 , 5 4 4}$ & $17,691,328$ \\
\hline \hline
\end{tabular}

Bold text highlights better performance. $D_{c}$ : Cosine distance.

The speaker verification dataset is from the VoxCeleb1 verification dataset. It contains 40 speakers that have IDs from 270-309. During the verification testing process, two audios are 

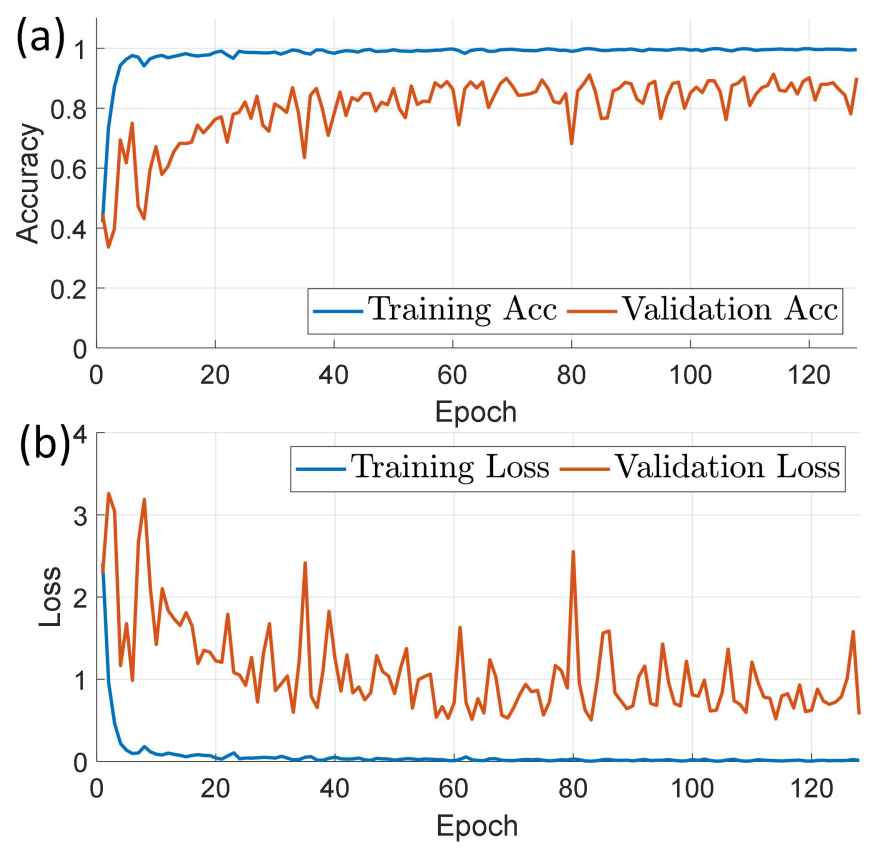

Figure 6.4: (A) Modified MobileNetV1 Training and Validation Accuracy; (B) Modified MobileNetV1 Training and Validation Loss
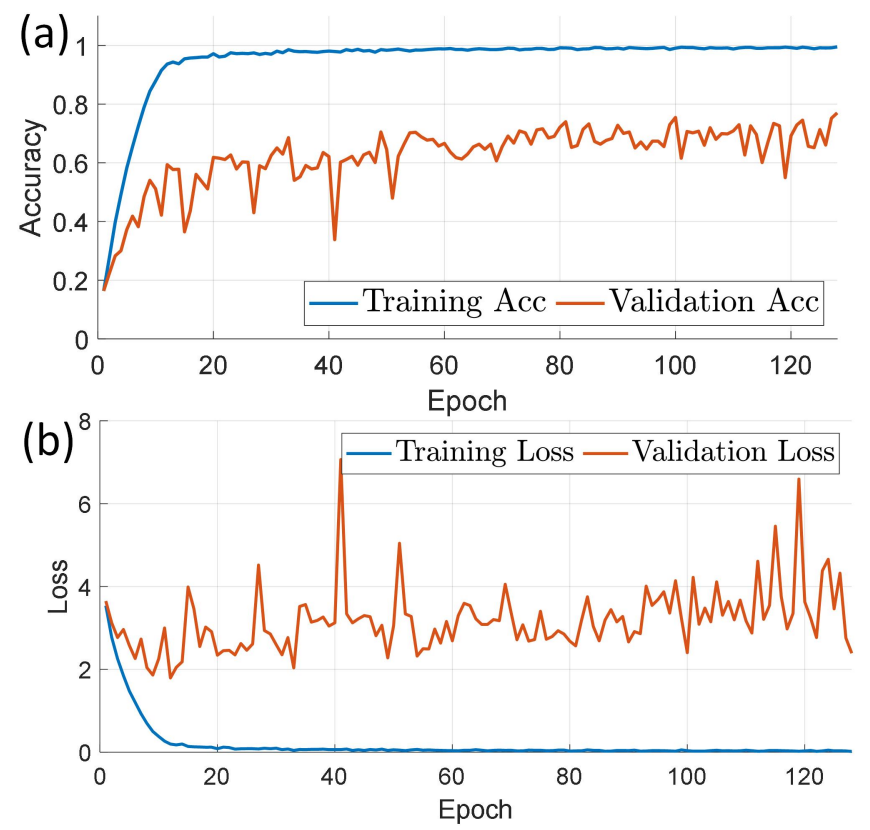

Figure 6.5: (A) VGG-M Training and Validation Accuracy; (B) VGG-M Training and Validation Loss 
given: one is used as enrolled data, and the other is used as test data. The verification test data is randomly divided into 3 segments each consisting of a 3 -second audio. The enrolled data is randomly divided into 3 segments each consisting of 3-second audio. The time cost to process one clip of 3-second audio will be measured to compare the performance.

\subsection{CVASV HMI}

The previous experiment uses the VoxCeleb1 dataset to train the neural network and test the speaker verification subsystem. The performance of using MobileNetV1 is now compared to the original method, which uses VGG-M. Using Mobile Net reduces the latency by $17.6 \%$ while maintaining similar verification accuracy. This result proves that the speaker verification system works as designed. However, the experiment is not entirely complete. The complete CVASV HMI is not tested using proper data. The data used in the experiment is from the VoxCeleb1 data set, which consists of arbitrary videos from YouTube. The data is suitable for training a robust speaker verification system. But the RML glove is designed for patients with hand disability to perform Activities of Daily Living(ADL). The application requires some specific commands, and the voice input tends to be short, with only 2 to 3 keywords. For example, phrases may include "grasp toothbrush", "grasp a bottle", "grasp cup", etc. There are not any public voice data sets designed specifically for this purpose. For this reason, the RML Exoskeleton Voice Control dataset was created to fulfill the special requirement of testing the RML exoskeleton glove. The dataset can be easily extended to more command and more speakers. 


\subsubsection{RML Exoskeleton Voice Control Dataset}

This dataset contains ten-speaker models. The ten speakers contain six males and four females, including Asian accents, Indian accents, and native North American English speakers. The structure of the speaker model is shown in Fig.6.6. Each speaker model is made up of 2 sections: an enrollment section and a testing section. The enrollment section contains 5 commands as enrollment data: "hey glove", "grasp bottle", "grasp cup", "grasp toothbrush", and "release". The enrollment data is recorded when each speaker reads these commands in a quiet room with normal speed and tone. This section is used to enroll the speaker and tune the CVASV HMI. The testing section contains 20-25 short commands for each speaker, recorded with various background noise. This section is used to test the performance of the CVASV HMI. This dataset can be used on other voice command systems with similar design principles as well.

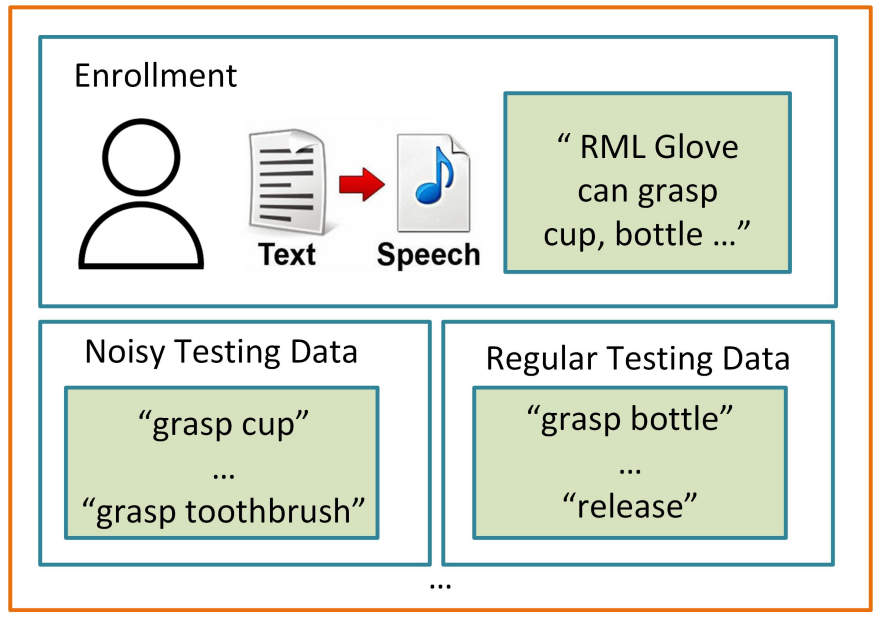

Figure 6.6: Structure of the Speaker Model of the RML Exoskeleton Voice Control Dataset 


\subsubsection{CVASV HMI Performance}

The RML Exoskeleton Voice Control data set (RML dataset) is used to test the CVASV HMI. The CVASV HMI is configured to use Google API with tuning for the recognition section. The verification section uses MobileNetv1 as a feature extraction network and uses a cosine distance threshold of alpha $=0.25$. During the enrollment, each speaker has five enrolled models which represent each different command. The voice command is first recognized and classified into five categories based on the command and then verified with the enrolled model under each category.

The first experiment was conducted to test the accuracy of each speaker. The enrollment model in the RML dataset is used to enroll each speaker. Within each speaker model, all the short commands are tested in two parts: recognition and verification. Each command is tested against its enrollment model. If the recognition result with tuning differs from the intended command, the recognition is marked as failed. If the verification section has a cosine distance larger than the 0.25 threshold, the verification is marked as failed. The result is shown in Tab. 6.3. The result proved that the CVASV has $89 \%$ accuracy. However, there is a significantly lower accuracy in recognition (89\%) than verification (97.5\%). The low recognition accuracy is caused by the Google API, which has a higher error rate than proposed.

The second experiment tested the latency of the system. The CVASV HMI is designed to reliably run in real-time on a portable device. To simulate the portable device, this experiment was done using a computer with a $2.2 \mathrm{GHz}$ six-core Intel i7 processor with CPU performance limited to $20 \%$ and RAM limited to 2 Gigabyte. The latency for each component is shown in Tab. 6.4. The entire system's latency is $182.58 \mathrm{~ms}$, which is faster than most HMIs and can run in real-time on a portable device. 
Table 6.3: CVASV HMI Accuracy Test

\begin{tabular}{c|c|c}
\hline \hline Speaker & Recognition Accuracy & Verification Accuracy \\
\hline A & $90 \%$ & $95 \%$ \\
B & $100 \%$ & $100 \%$ \\
C & $70 \%$ & $100 \%$ \\
D & $90 \%$ & $100 \%$ \\
E & $95 \%$ & $100 \%$ \\
F & $75 \%$ & $95 \%$ \\
G & $85 \%$ & $95 \%$ \\
H & $100 \%$ & $100 \%$ \\
I & $90 \%$ & $95 \%$ \\
J & $95 \%$ & $95 \%$ \\
\hline \hline
\end{tabular}

Table 6.4: Time Cost to process a one audio command

\begin{tabular}{c|c}
\hline \hline & Time Cost \\
\hline Noise Reduction Filter & $2.12 \mathrm{~ms}$ \\
Loudness Filter $\left(D_{e}\right)$ & $2.45 \mathrm{~ms}$ \\
Audio Collection Subsystem & $5.02 \mathrm{~ms}$ \\
Spectrum Conversion & $12.32 \mathrm{~ms}$ \\
Voice Recognition API & $34.47 \mathrm{~ms}$ \\
MobileNetV1 Feature extractor & $126.2 \mathrm{~ms}$ \\
Voice Recognition Subsystem & $182.58 \mathrm{~ms}$ \\
\hline \hline
\end{tabular}

Table 6.5: Influence of Background Noise on CVASV HMI

\begin{tabular}{c|c|c}
\hline \hline command & Noisy Dis & Quiet Dis \\
\hline "hey glove" & 0.17 & 0.12 \\
"grasp toothbrush" & 0.13 & 0.11 \\
"grasp bottle" & 0.12 & 0.06 \\
"grasp cup" & 0.12 & 0.09 \\
"release" & 0.21 & 0.13 \\
\hline \hline
\end{tabular}

The third experiment was conducted to study the impact of noisy data on CVASV HMI. The same command of the same speaker under different background noise is compared. The results are shown in Tab. 6.5. The verification has a lower accuracy in its noisy environment. However, all the data tested in the noisy environment performs under the 0.25 threshold. It proves that the system performs well under noisy environment.

The forth experiment was conducted to study how biological gender influences the verification system. Speaker A and speaker B are of opposite sex. Speaker A is enrolled and tested 
with speaker B's testing data. Theoretically, none of these commands will be accepted. Speaker A is then enrolled and tested with speaker C's testing data, where Speakers A and $\mathrm{C}$ are of the same sex. Theoretically, none of these commands should be accepted either. The results of this experiment are shown in Tab. 6.6.

Table 6.6: Influence of Gender on CVASV HMI

\begin{tabular}{c|c|c}
\hline \hline command & Same Sex Dis & Oppos. Sex Dis \\
\hline "hey glove" & 0.52 & 0.48 \\
"grasp bottle" & 0.37 & 0.39 \\
"grasp cup" & 0.47 & 0.42 \\
"grasp toothbrush" & 0.44 & 0.39 \\
"release" & 0.28 & 0.51 \\
\hline \hline
\end{tabular}

When performing verification over speakers with the same and opposite sex, there were no significant differences between the two scenarios. All the test data performed above the 0.25 distance threshold as expected.

The fifth experiment was done to study how accent will affect the CVASV HMI. Speaker E and Speaker C are of the same gender. Speaker E is a native English speaker without a noticeable accent. Speaker $\mathrm{C}$ is a fluent English speaker with a noticeable accent. From Tab. 6.3, a speaker with an accent will have lower accuracy in the recognition system, but the verification system is not affected. In this experiment, verification accuracy was tested. Speaker E is enrolled and tested against speaker B's testing data. Theoretically, none of these commands will be accepted. The result is shown in Tab. 6.7. The results proved that accent does not affect the verification system. All distance values are higher than the 0.25 threshold as expected.

The sixth experiment tested whether the speaker verification can verify the same speaker with a genuine verification model. As mentioned previously, for each speaker, each command has its enrollment model. This experiment was performed to verify whether using a genuine model for all commands is actually feasible. For speaker A, all the enrollment commands are 
Table 6.7: Influence of Accent on CVASV HMI

\begin{tabular}{c|c}
\hline \hline command & Distance \\
\hline "hey glove" & 0.32 \\
"grasp toothbrush" & 0.34 \\
"grasp bottle" & 0.42 \\
"grasp cup" & 0.38 \\
"release" & 0.47 \\
\hline \hline
\end{tabular}

combined and enrolled as a genuine model. All test data was tested against this command. The results are shown in Tab. 6.8. The same speaker saying different commands has a similar physical distance to different speakers saying the same command. The commands are too short to distinguish between similar voices. To use a genuine model to perform speaker verification, the command needs to be made longer. However, using a longer command is not practical. The current solution is to have a separate model for each speaker containing every command. Using a separate model for each command and speaker will allow the speaker verification network to distinguish between different speakers.

Table 6.8: Cosine distance between different commands of same speaker

\begin{tabular}{c|c}
\hline \hline command & distance \\
\hline "hey glove" & 0.34 \\
"grasp toothbrush" & 0.42 \\
"grasp bottle" & 0.33 \\
"grasp cup" & 0.33 \\
"release" & 0.33 \\
\hline \hline
\end{tabular}

\subsection{Personalized Voice Activated Grasping System}

An experiment of the Personalized Voice Activated Grasping System was performed on the SEAs. This experiment uses a computer to simulate a smartphone to act as a high-level control unit. The experiment includes using the CVASV HMI to initialize the grasp, using SEA to apply proper force, and using slip detection to maintain the grasp's stability. The 


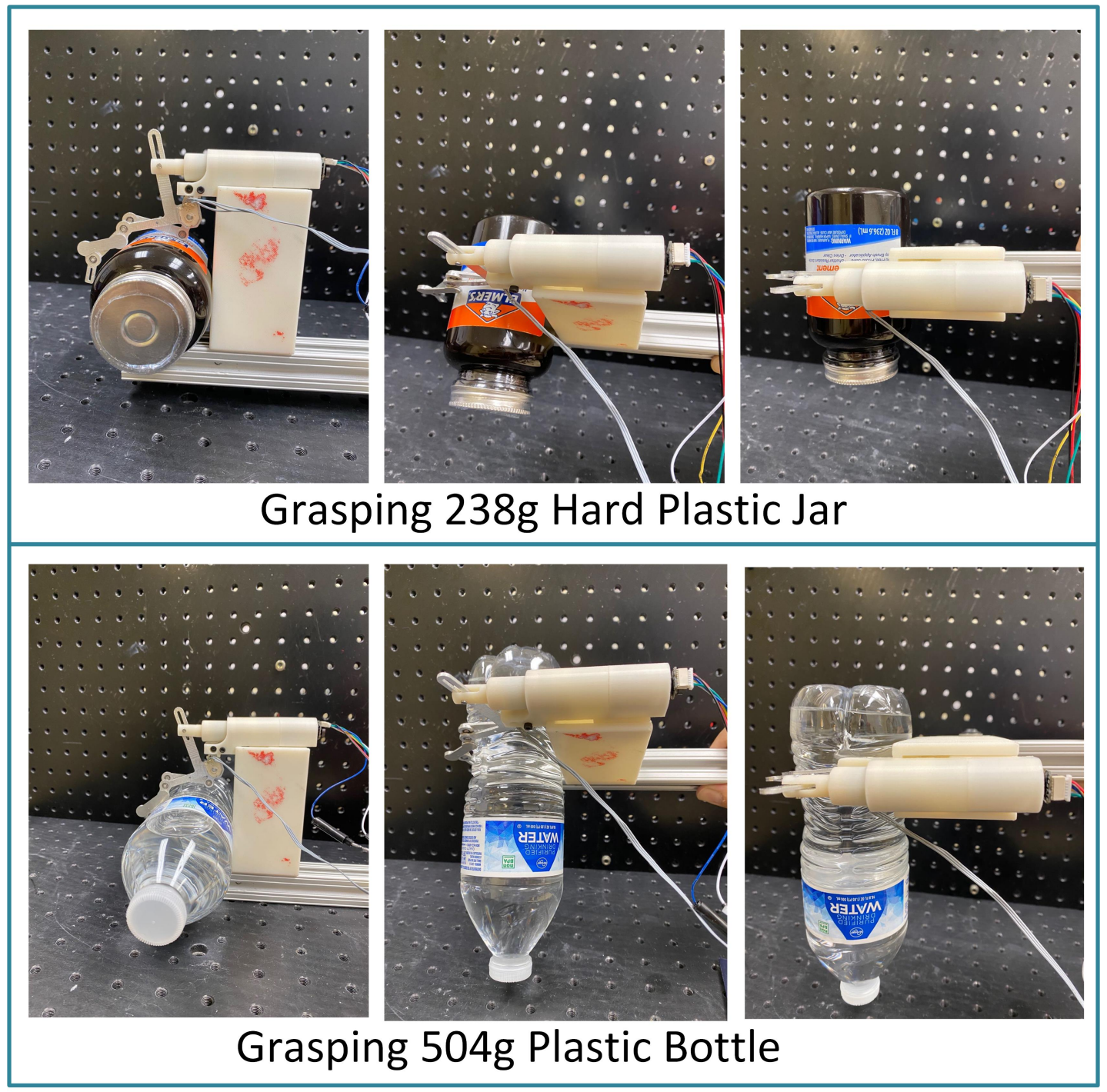

Figure 6.7: Grasping with Personalized Voice Activated Grasping System using Linear SEA

result is shown in Fig. 6.7 and Fig. 6.8. The CVASV HMI successfully initiated the grasp. The electronics and low-level control functioned flawlessly to grasp a heavy object. When the angle changes, the slip detection algorithm can detect slip and apply additional force incrementally to maintain a stable grasp. The latency between inputting a voice command to the glove and initiating a grasp is $184.58 \mathrm{~ms}$. This latency proved the CVASV HMI, and the low-level control can run in real-time with low latency. 

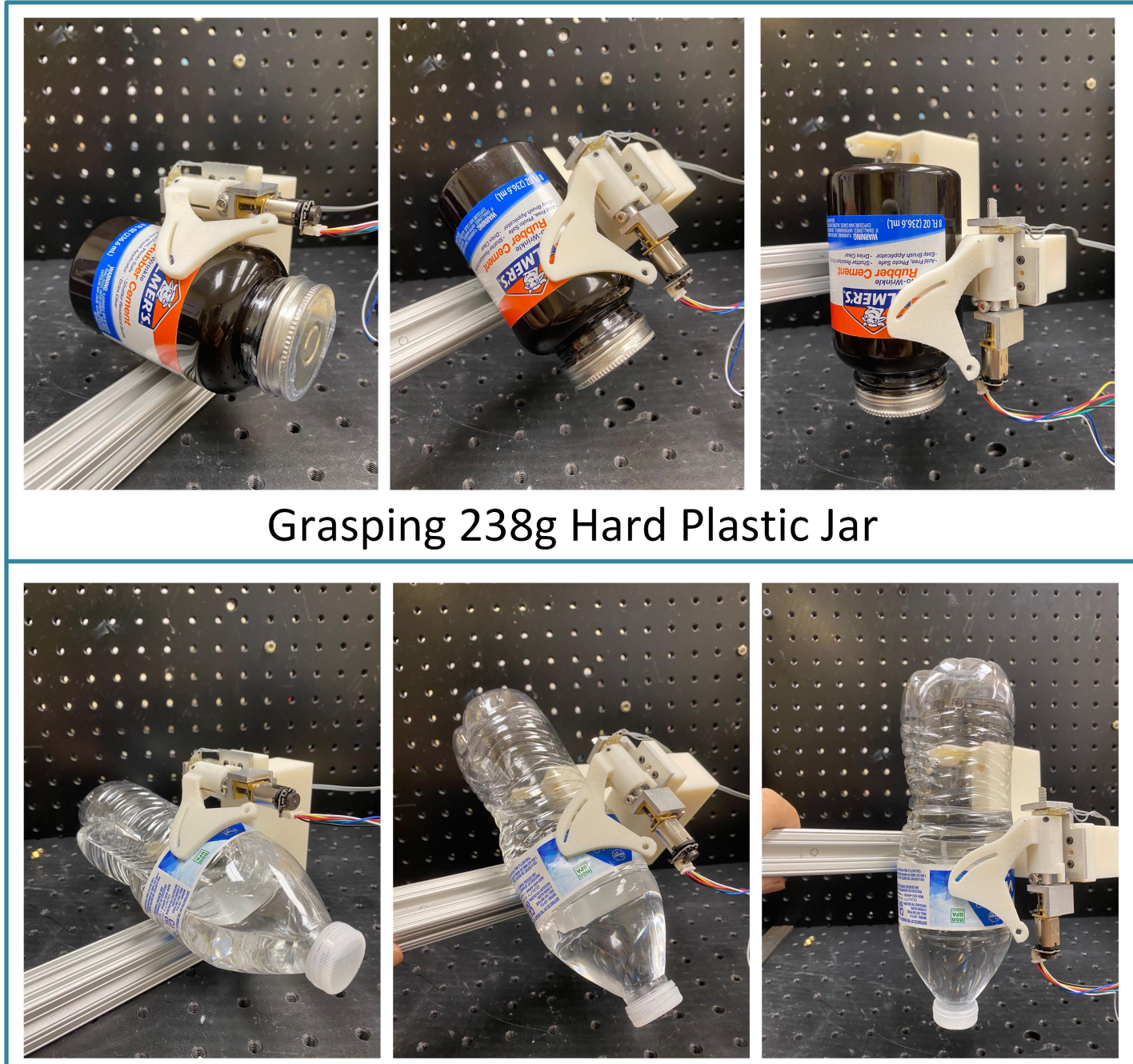

Grasping 301g Plastic Bottle

Figure 6.8: Grasping with Personalized Voice Activated Grasping System using Rotatory SEA 


\section{Chapter 7}

\section{Conclusion and Future Work}

\subsection{Conclusion}

The Personalized Voice Activated Grasping System proposed in this research was proven to be fast, accurate, portable, and a secure method to control the RML glove through voice activation. The electronics and hardware were able to function without issues for more than 100 hours of testing and proved to be reliable. The functionality was complete and was able to provide force feedback, force control, and connectivity. The electronics and hardware performed well and did not require any improvement. The low-level control system was fast and accurate. The force feedback SEAs were accurate and could detect slip. The CVASV HMI can distinguish between different speakers and recognize different commands. The entire system has less than 200ms latency and has an average accuracy of $92.5 \%$ command acceptance.

The CVASV HMI provided an extra layer of security. This speaker verification can eliminate the chance of picking up false commands from background noise and decreasing the exoskeleton's chance of being mis-manipulated. However, it does not provide high-level security. Recorded audio or a similar voice will easily defect this system.

The Personalized Voice Activated Grasping System will be further tested on the RML glove after the glove is finished. The RML exoskeleton dataset will be extended to 50 speakers 
with data collected from real patients' clinical trials. With the data from actual patients, the system can be modified to assist patients.

The CVASV HMI will be applied on a smartphone and tested with a larger dataset. If the recognition accuracy is not satisfactory during further testing, the Google API will be removed, and a deep learning-based short-command recognition system will be used to increase the recognition accuracy. The Personalized Voice Activated Grasping System will also be tested with patients to improve the user interface.

\subsection{Future Work}

\subsubsection{Electronics and Hardware}

The experiments on a single finger proved that the electronics and hardware fulfill all the requirements. The entire glove needs to be manufactured and assembled to enable further testing of the complete system.

\subsubsection{CVASV Recognition Subsystem}

Some improvements can be pursued in the future. According to the experiments, the google API has a very high word-error rate, making the system to require significant similar word tuning. The Google API which used the current CVASV HMI sometimes failed to recognize the command and there appears to be room for improvement.

The advantage of using Google API is that it is a robust system which is fast and can be quickly adapted to different languages and commands. However, Google API has a significantly higher word-error rate than what they proposed and can be easily affected by 
a foreign accent. With the unexpected high word-error rate, most commands need ample similar word tuning, which reduced the robustness of using google API.

Google API is fast but requires an internet connection to perform cloud computing. Connection to the internet is crucial to the CVASV HMI, which makes it less portable. There might be situations where the patient does not have internet access. In the future, it is necessary to replace the Google API with a more advanced voice recognizer. The current CVASV HMI only has less than 200ms delay. Therefore, adding another 100ms delay to gain more accuracy would be acceptable. A MobileNetV1 based voice recognizer can replace the Google API based voice recognizer.

A normal voice recognizer needs to be robust. Google voice recognizer [28] and Prerana et al's approach [29] are good examples of deep learning-based voice recognizers. Those approaches need to be able to recognize random words and distinguish similar words. For example, it needs to differentiate between "grasp", "crest" and "grass." However, when the data is noisy or the speaker has a strong accent, it cannot recognize the correct word. A command system is different from a normal voice recognizer in a manner such that a command system will only need to take a few input combinations, and these word combinations can be specifically designed to avoid ambiguity.

To replace the google API in the CVASV HMI, a deep learning-based, short phrase recognizer can be used. The new recognizer can be directly trained to classify different commands. However, these might reduce the robustness due to the limitation of data. The recognizer can use the feature extraction layer of a normal voice recognizer to extract important features. Using KNN, modeled KNN [30], or GMM [31] would be required to perform classification. All input commands can be classified into several commands, and as such will reduce the ambiguity. 


\subsubsection{CVASV Verification Subsystem}

The verification system achieves a $97.25 \%$ accuracy when tested with the RML voice dataset. The deep learning network is proven to be very accurate. However, there are still some improvements that can be made. Nangrani et al. [22] has proven that using large margin Softmax loss function [32], and adding NetVALD [33] or GhostVALD [34] will improve the accuracy of VGG-M. The CVASV HMI is designed based on Nangriani et al. [22] work. A similar method could be used to improve accuracy.

Extending the training dataset to VoxCeleb2 [35] should improve the accuracy without any detrimental effects. 


\section{Bibliography}

[1] E. M. Refour, B. Sebastian, R. J. Chauhan, and P. Ben-Tzvi, "A General Purpose Robotic Hand Exoskeleton With Series Elastic Actuation," Journal of Mechanisms and Robotics, vol. 11, no. 6, 2019.

[2] T. Vanteddu, B. Sebastian, and P. Ben-Tzvi, "Design optimization of RML glove for improved grasp performance," in Proceedings of the ASME 2018 Dynamic Systems and Control Conference, DSCC 2018, vol. 1, pp. 1-8, 2018.

[3] Z. Ma, P. Ben-Tzvi, and J. Danoff, "Modeling human hand and sensing hand motions with the fivefingered haptic glove mechanism," in Proceedings of the 2014 ASME Int'l Design Engineering Technical Conferences $\&$ Computers and Information in Engineering Conf. (IDETC/CIE 2014), 38th Mechanisms \& Robotics Conference, (Buffalo, NY), 2014.

[4] Z. Ma and P. Ben-Tzvi, "RML glove-an exoskeleton glove mechanism with haptics feedback," IEEE/ASME Transactions on Mechatronics, vol. 20, no. 2, pp. 641-652, 2015.

[5] Z. Ma and P. Ben-Tzvi, "Tendon transmission efficiency of a two-finger haptic glove," in Proceedings of the ROSE 2013 - 2013 IEEE International Symposium on Robotic and Sensors Environments Conference, (Washington, DC), 2013.

[6] Z. Ma and P. Ben-Tzvi, "Design and optimization of a five-finger haptic glove mechanism," Journal of Mechanisms and Robotics, vol. 7, no. 4, pp. 1-8, 2015.

[7] Z. Ma, P. Ben-Tzvi, and J. Danoff, "Hand Rehabilitation Learning System with an 
Exoskeleton Robotic Glove," IEEE Transactions on Neural Systems and Rehabilitation Engineering, vol. 24, no. 12, pp. 1323-1332, 2016.

[8] Z. Ma, P. Ben-tzvi, and S. Member, "Sensing and Force-Feedback Exoskeleton ( SAFE ) Glove," in Proceedings of the 2015 ASME IDETC/CIE, 39th Mechanisms \& Robotics Conference, (Boston, MA), 2015.

[9] B. J. B. Lee, A. Williams, P. Ben-tzvi, and S. Member, "for Rehabilitation and Assistive Applications," IEEE Transactions on Neural Systems and Rehabilitation Engineering, vol. 26 , no. 8, pp. 1556-1565, 2018.

[10] J. Lee and P. Ben-Tzvi, "Design of a Wearable 3-DOF Forearm Exoskeleton for Rehabilitation and Assistive Purposes," in Proceedings of the 2017 ASME International Mechanical Engineering Congress and Exposition Conference (IMECE 2017), (Tampa, Florida), 112017.

[11] E. Refour, B. Sebastian, and P. Ben-Tzvi, "Two-digit robotic exoskeleton glove mechanism: Design and integration," Journal of Mechanisms and Robotics, vol. 10, no. 2, pp. 1-9, 2018.

[12] E. Refour, B. Sebastian, and P. Ben-Tzvi, "Design and Implementation of an Exoskeleton Glove for Infant Medical Rehabilitation," in Proceedings of the 2018 ASME IDETC/CIE, 42nd Mechanisms \& Robotics Conference, (Quebec City, Canada), 82018.

[13] R. J. Chauhan and P. Ben-Tzvi, "A Series Elastic Actuator Design and Control in a Linkage Based Hand Exoskeleton," in Proceedings of the ASME 2019 Dynamic Systems and Control Conference (DSCC 2019), (Park City, Utah), 102019.

[14] R. Chauhan, B. Sebastian, S. Member, P. Ben-tzvi, and S. Member, "Exoskeleton Glove 
Control," IEEE Transactions on Human-Machine Systems, vol. PP, no. 1, pp. 1-10, 2019.

[15] R. J. Chauhan and P. Ben-Tzvi, "Latent Variable Grasp Prediction for Exoskeletal Glove Control," in Proceedings of the ASME 2018 Dynamic Systems and Control Conference (DSCC 2018), (Atlanta, GA), 92018.

[16] T. Vanteddu and P. Ben-Tzvi, "Stable Grasp Control with a Robotic Exoskeleton Glove," Journal of Mechanisms and Robotics, vol. 12, no. 6, pp. 1-14, 2020.

[17] W. Xu, S. Pradhan, Y. Guo, B.-T. Pinhas, and C. Bravo, "A Novel Design of A Robotic Glove System For Paitients with Brachial Plexus Injuries," in Proceedings of the 2020 ASME IDETC/CIE, 44th Mechanisms $\&$ Robotics Conference, (St. Louis, MO), 2020.

[18] T. Feix, R. Pawlik, H.-B. Schmiedmayer, J. Romero, and D. Kragi, "A comprehensive grasp taxonomy," in Proceedings of the Robotics, Science and Systems Conference: Workshop on Understanding the Human Hand for Advancing Robotic Manipulation, pp. 2-3, 2009 .

[19] L. Randazzo, I. Iturrate, R. Chavarriaga, R. Leeb, and J. D. R. Millan, "Detecting intention to grasp during reaching movements from EEG," in Proceedings of the Annual International Conference of the IEEE Engineering in Medicine and Biology Society, EMBS, vol. 2015-Novem, pp. 1115-1118, 2015.

[20] D. Kim, B. B. Kang, K. B. Kim, H. Choi, J. Ha, K.-J. Cho, and S. Jo, "Eyes are faster than hands: A soft wearable robot learns user intention from the egocentric view," Science Robotics, vol. 4, no. 26, p. eaav2949, 2019.

[21] X. Wang, P. Tran, S. M. Callahan, S. L. Wolf, and J. P. Desai, "Towards the development of a voice-controlled exoskeleton system for restoring hand function," in Proceedings of 
the 2019 International Symposium on Medical Robotics(ISMR) Conference, (Atlanta, GA), pp. 1-7, IEEE, 2019.

[22] A. Nagrani, J. S. Chung, W. Xie, and A. Zisserman, "Voxceleb: Large-scale speaker verification in the wild," Computer Speech and Language, vol. 60, p. 101027, 32020.

[23] A. G. Howard, M. Zhu, B. Chen, D. Kalenichenko, W. Wang, T. Weyand, M. Andreetto, and H. Adam, "MobileNets: Efficient Convolutional Neural Networks for Mobile Vision Applications," arXiv abs/1704.04861, 42017.

[24] J. B. Lee, "Development of Intelligent Exoskeleton Grasping Through Sensor Fusion and Slip Detection Development of Intelligent Exoskeleton Grasping," Master's thesis, Virginia Polytechnic Institute and State University, 2018.

[25] J. Sueur, A very short introduction to sound analysis for those who like elephant trumpet calls or other wildlife sound. 2014.

[26] M. D. Balasingam and C. S. Kumar, "Refining Cosine Distance Features for Robust Speaker Verification," in Proceedings of the 2018 IEEE International Conference on Communication and Signal Processing, ICCSP 2018, no. 1, pp. 152-155, 2018.

[27] A. Nagraniy, J. S. Chungy, and A. Zisserman, "VoxCeleb: A large-scale speaker identification dataset," Proceedings of the Annual Conference of the International Speech Communication Association, INTERSPEECH, vol. 2017-Augus, pp. 2616-2620, 2017.

[28] C. C. Chiu, T. N. Sainath, Y. Wu, R. Prabhavalkar, P. Nguyen, Z. Chen, A. Kannan, R. J. Weiss, K. Rao, E. Gonina, N. Jaitly, B. Li, J. Chorowski, and M. Bacchiani, "State-of-the-art speech recognition with sequence-to-sequence models," in Proceedings of the 2018 IEEE International Conference on Acoustics, Speech and Signal Processing (ICASSP), pp. 4774-4778, 2018. 
[29] G. C. Hansen, K. H. Falkenbach, and I. Yaghmai, "Voice recognition system.," in Proceedings of the Journal of Applied and Fundamental Sciences signal, vol. 169, p. 580, 2015.

[30] G. Guo, H. Wang, D. Bell, Y. Bi, and K. Greer, "KNN model-based approach in classification," Lecture Notes in Computer Science (including subseries Lecture Notes in Artificial Intelligence and Lecture Notes in Bioinformatics), vol. 2888, pp. 986-996, 2003.

[31] R. Chellappa, A. Veeraraghavan, N. Ramanathan, C.-Y. Yam, M. S. Nixon, A. Elgammal, J. E. Boyd, J. J. Little, N. Lynnerup, P. K. Larsen, and D. Reynolds, "Gaussian Mixture Models," Encyclopedia of Biometrics, no. 2, pp. 659-663, 2009.

[32] W. Liu, Y. Wen, Z. Yu, and M. Yang, "Large-Margin Softmax Loss for Convolutional Neural Networks," in Proceedings of the 33rd International Conference on International Conference on Machine Learning - Volume 48, (New York, NY, USA), p. 507-516, JMLR.org, 2016.

[33] R. Arandjelovic, P. Gronat, A. Torii, T. Pajdla, and J. Sivic, "NetVlAD: CNN Architecture for Weakly Supervised Place Recognition," IEEE Transactions on Pattern Analysis and Machine Intelligence, vol. 40, no. 6, pp. 1437-1451, 2018.

[34] Y. Zhong, R. Arandjelović, and A. Zisserman, "GhostVLAD for Set-Based Face Recognition," Lecture Notes in Computer Science (including subseries Lecture Notes in Artificial Intelligence and Lecture Notes in Bioinformatics), vol. 11362 LNCS, pp. 35-50, 2019.

[35] J. S. Chung, A. Nagrani, and A. Zisserman, "VoxceleB2: Deep speaker recognition," in Proceedings of the Annual Conference of the International Speech Communication Association, INTERSPEECH, vol. 2018-Septe, pp. 1086-1090, 2018. 